\title{
Detailed Exploration of Face-related Processing in Congenital Prosopagnosia: 1. Behavioral Findings
}

\author{
Marlene Behrmann ${ }^{1}$, Galia Avidan', \\ Jonathan J. Marotta ${ }^{2}$, and Rutie Kimchi ${ }^{3}$
}

\begin{abstract}
We show that five individuals with congenital prosopagnosia (CP) are impaired at face recognition and discrimination and do not exhibit the normal superiority for upright over inverted faces despite intact visual acuity, low-level vision and intelligence, and in the absence of any obvious neural concomitant. Interestingly, the deficit is not limited to faces: The CP individuals were also impaired at discriminating common objects and novel objects although to a lesser extent than discriminating faces. The perceptual deficit may be attributable
\end{abstract}

\section{INTRODUCTION}

Congenital prosopagnosia (CP) refers to the lifelong impairment in face processing that is apparent from birth, despite intact visual and intellectual functions. The term "congenital" is used to denote the absence of a lesion or other neurological concomitant acquired at any stage of development (Jones \& Tranel, 2001), and excludes individuals suffering from visual deprivation, as in cases of infantile cataracts, or from other developmental problems such as autism. CP also contrasts with the more general term "developmental prosopagnosia," which includes CP as well as individuals who have sustained brain damage before birth or in early childhood (Barton, Cherkasova, Press, Intriligator, \& O'Connor, 2003; Farah, Rabinowitz, Quinn, \& Liu, 2000). $\mathrm{CP}$ can be severely debilitating, affecting the recognition of even the most familiar individuals (Nunn, Postma, \& Pearson, 2001). These CP individuals may also fail to discriminate between two unknown faces, usually recognizing others by voice or other cues such as clothing or accessories. As in acquired prosopagnosia (AP), CP individuals are typically able to acknowledge that a face is present but are unable to assign identity to the face. Unlike AP, however, CP may go undetected as the individual has no means of comparison with normal face processing skills. In the last couple of years, there has been increasing interest in CP (e.g., Behrmann \&

\footnotetext{
${ }^{1}$ Carnegie Mellon University, Pittsburgh, ${ }^{2}$ University of Manitoba, Winnipeg, ${ }^{3}$ University of Haifa
}

to a more fundamental visual processing disorder; the $\mathrm{CP}$ individuals exhibited difficulty in deriving global configurations from simple visual stimuli, even with extended exposure duration and considerable perceptual support in the image. Deriving a global configuration from local components is more critical for faces than for other objects, perhaps accounting for the exaggerated deficit in face processing. These findings elucidate the psychological mechanisms underlying CP and support the link between configural and face processing.

Avidan, 2005; Duchaine \& Nakayama, 2005; Kress \& Daum, 2003a). However, many findings remain inconsistent, perhaps as a function of the paucity of cases, the apparent heterogeneity of the disorder and/or the varying methods of assessment.

Although an impairment in face processing is de facto required for the diagnosis of $\mathrm{CP}$, face processing is not monolithic and can involve detecting the presence of a face, discriminating between two faces or identifying an individual face. Which of these processes is implicated in $\mathrm{CP}$ is unclear; although some CP individuals succeed in relatively easy matching tasks (perhaps by matching individual features), when reaction time (RT) is measured and task demands are increased, the deficit is uncovered (Kress \& Daum, 2003a). The extent to which CP individuals can recognize famous faces has also yielded mixed results: some perform poorly (de Gelder \& Rouw, 2000; Bentin, Deouell, \& Soroker, 1999), whereas others perform quite well (Duchaine \& Nakayama, 2005; Duchaine, 2000; Temple, 1992). Note that the images used in these tests often contain salient cues such as clothing; because $\mathrm{CP}$ individuals are particularly adept at exploiting such cues, these latter cases may compensate for the underlying recognition deficit. The heterogeneity of findings clearly calls for a systematic analysis of face processing in $\mathrm{CP}$.

A second relevant issue, which remains the subject of an ongoing controversy, is the extent to which prosopagnosia (AP and $\mathrm{CP}$ ) is specific to faces. Neuropsychological studies have demonstrated a double dissociation between the recognition of faces and objects 
(Moscovitch, Winocur, \& Behrmann, 1997; McNeil \& Warrington, 1993), and functional imaging (Kanwisher, McDermott, \& Chun, 1997), ERP (Bentin, Allison, Puce, Perez, \& McCarthy, 1996), and physiology (Rolls, 1984; Perrett, Rolls, \& Caan, 1979) studies point to the existence of a neural system specialized, if not dedicated, to faces (Behrmann \& Moscovitch, 2001). An alternative view, which also has widespread support, is that there is a general-purpose visual process that subserves all visual objects and the dissociations arise because of the unusual demands placed on the system by faces (Tarr \& Cheng, 2003). Because face processing typically involves individual identification, whereas other objects are usually recognized at a basic level (e.g., as a chair or an apple), faces engage fine-grained discrimination of perceptually similar exemplars within a category and individuals become expert at these discriminations. Whether recognition of other visual nonface objects is also impaired when homogeneous, complex stimuli are employed remains debatable. Many AP individuals have difficulties categorizing exemplars of within-class objects, which share the same complex configuration (Gauthier, Behrmann, \& Tarr, 1999; De Haan and Campbell, 1991; Etcoff, Freeman, \& Cave, 1991; Damasio, Damasio, \& Hoesen, 1982), although this does not seem to be true for all cases (Farah, Wilson, Drain, \& Tanaka, 1995; Sergent \& Signoret, 1992; De Renzi, Faglioni, Grossi, \& Nichelli, 1991).

In a review of nine cases of $\mathrm{CP}$, two are impaired at recognizing nonface objects, one mildly and one severely (Kress \& Daum, 2003a) and, in a recent study, using an old/new recognition memory paradigm, four out of seven CP individuals showed a dissociation between memory for faces and objects (Duchaine \& Nakayama, 2005). We note, however, that the methods of assessment vary considerably across studies and that reaction time is generally not measured despite the possibility that the $\mathrm{CP}$ individuals might be inordinately slow or might even trade speed off against accuracy. To explore the specificity of the deficit, then, we examine the performance of the CP individuals in tasks using common objects and novel objects under conditions where the task demands and measurements are equated, as closely as possible, to those required for face discrimination.

The final major issue concerns the relationship between face processing and configural perception. Faces form a class of perceptually similar visual stimuli and are, therefore, thought to be the paradigmatic example of a stimulus that relies on configural processing with the holistic properties of the stimulus overriding the contribution of its individual components (Maurer, Le Grand, \& Mondloch, 2002; Farah, Wilson, Drain, \& Tanaka, 1995; Tanaka \& Farah, 1993). It has also been suggested that deriving the second-order statistics, or the spatial relations between the components, is particularly critical for face processing (Carey \& Diamond, 1994; Rhodes,
1988). The failure to integrate visual elements is thought to underlie prosopagnosia (Barton, Press, Keenan, \& O'Connor, 2002; Levine \& Calvanio, 1989), with the result that these patients rely on a more piecemeal or feature-based strategy in constructing face representations. Although the failure to derive configurations is thought to be particularly devastating for face processing, given the homogeneity between exemplars and the need to rely on the second-order statistics of the input (Rhodes, 1988), a configural impairment may affect other nonface stimuli, too, if the spatial relations between the components are required to differentiate perceptually similar exemplars.

The purpose of the current article is to explore these three issues in a group of five adults with CP. Our findings show that all CP subjects are impaired at face recognition and discrimination, and do not show the well-known benefit for upright over inverted faces. Interestingly, all of these same individuals are impaired at nonface object recognition, although this is much more variable and less severe than their face processing difficulty. Finally, all subjects are impaired at configural processing, exhibiting difficulty in deriving the global configuration of even very simple visual images, letters, and geometric shapes. The apparent failure to extract the spatial relations between the local elements is conjectured to contribute to the disproportionate failure to process faces, relative to other visual stimuli.

\section{RESULTS}

A group of five $\mathrm{CP}$ individuals participated in three series of studies, each addressing one of the issues laid out above. In all five individuals, $\mathrm{CP}$ was manifested without any obvious underlying neurological cause, and visual acuity and basic visual processing abilities were unimpaired (see Table 1 for biographical details and results of low-level visual tasks). We compared the performance

Table 1. Congenital Prosopagnosic Subjects

\begin{tabular}{lcccc}
$\begin{array}{l}\text { CP Subjects' } \\
\text { Initials }\end{array}$ & Sex & Age & $\begin{array}{c}\text { Log Contrast } \\
\text { Thresholds }^{\mathrm{a}}\end{array}$ & $\begin{array}{c}\text { Gabor Contour } \\
\text { Detection } \Delta^{\mathrm{b}}\end{array}$ \\
\hline $\mathrm{TM}$ & $\mathrm{M}$ & 27 & normal & .65 \\
$\mathrm{KM}$ & $\mathrm{F}$ & 60 & normal & .7 \\
$\mathrm{NI}$ & $\mathrm{M}$ & 40 & normal & .55 \\
MT & $\mathrm{M}$ & 41 & normal & .6 \\
$\mathrm{BE}$ & $\mathrm{F}$ & 29 & normal & .55 \\
\hline
\end{tabular}

${ }^{a}$ The thresholds were initially established for control subjects; any threshold for CP which exceeds 95\% confidence interval at any of the tested CPIs would be considered deviant. All CP subjects performed in the normal range for all CPIs.

bAverage $\Delta(\mathrm{CI})$ for controls: .65 (.07). Note that the lower the $\Delta$, the better the performance. All CP subjects performed within the normal range. 
Table 2. Control and AP Subjects

\begin{tabular}{|c|c|c|}
\hline Subjects' Initials & Sex & Age \\
\hline \multicolumn{3}{|c|}{ (i) Control Subjects } \\
\hline $\mathrm{SD}$ & $\mathrm{F}$ & 34 \\
\hline HM & $\mathrm{F}$ & 30 \\
\hline FN & M & 28 \\
\hline GT & M & 22 \\
\hline NU & $\mathrm{F}$ & 60 \\
\hline $\mathrm{DN}$ & $\mathrm{F}$ & 61 \\
\hline QN & $\mathrm{M}$ & 46 \\
\hline EQ & M & 40 \\
\hline $\mathrm{KM}$ & M & 36 \\
\hline UD & M & 44 \\
\hline \multicolumn{3}{|c|}{ (ii) Acquired Prosopagnosia } \\
\hline SM & M & 27 \\
\hline CR & M & 21 \\
\hline $\mathrm{RN}$ & M & 41 \\
\hline
\end{tabular}

of the CP subjects with that of a matched group of 12 control subjects (see Table 2 ). In addition, to provide a clear indication of the extent of the impairment in the CP individuals, we also compared the discrimination of face and of nonface stimuli of the CP group with that of three individuals with AP, sustained following brain damage (see Table 2).

\section{Face Discrimination and Recognition}

Because standardized tests of face processing have not proven effective in eliciting the disorder in prosopagnosia (Duchaine \& Nakayama, 2005; Galaburda \& Duchaine, 2003) and measures of accuracy alone are often considered insufficient (Delvenne, Seron, Coyette, \& Rossion, 2004; Gauthier et al., 1999), we tested the subjects on a series of customized face recognition and discrimination tasks, obtaining measures of RT, accuracy, and sensitivity $\left(A^{\prime}\right)$. Also, because the summary statistics (mean, $S D$ ) yielded by a small control group is usually not reliable enough to reflect population parameters (Crawford \& Garthwaite, 2002), we followed the procedure used by many researchers to assess abnormal performance in individual patients (Barton, Press, et al., 2002) and calculated the 95\% confidence interval from the control data.

The first set of tasks compared the $\mathrm{CP}$ and control subjects on the recognition of famous faces. On a set of 97 stimuli consisting of photographs of famous individuals (see Figure 1A), taken from popular magazines and from the Internet, the CP group identified significantly fewer faces than the controls $[F(1,15)=7.9, p<.01]$ (Figure 1B), with four of the five CP subjects (excluding TM) falling outside the $95 \%$ normal confidence inter-
Figure 1. Famous face experiments. (A) Examples of stimuli for Set 1 famous faces. (B) Mean \% (and $1 S E$ ) accuracy for correct identification of famous faces photographs for control and $\mathrm{CP}$ groups. Each symbol in the $\mathrm{CP}$ bars reflects the performance of one of the $\mathrm{CP}$ individuals and the same symbol is used for the same individual on all subsequent graphs. The dashed horizontal lines indicate the lower 95\% confidence interval calculated for the control group. (C) Examples of stimuli for Set 2 famous faces. (D) Performance (and $1 S E$ ) of the controls and $\mathrm{CP}$ individuals (and each $\mathrm{CP}$ individual) on Set 2 famous faces photographs. The dashed lines indicate the lower 95\% confidence interval calculated for the control group.

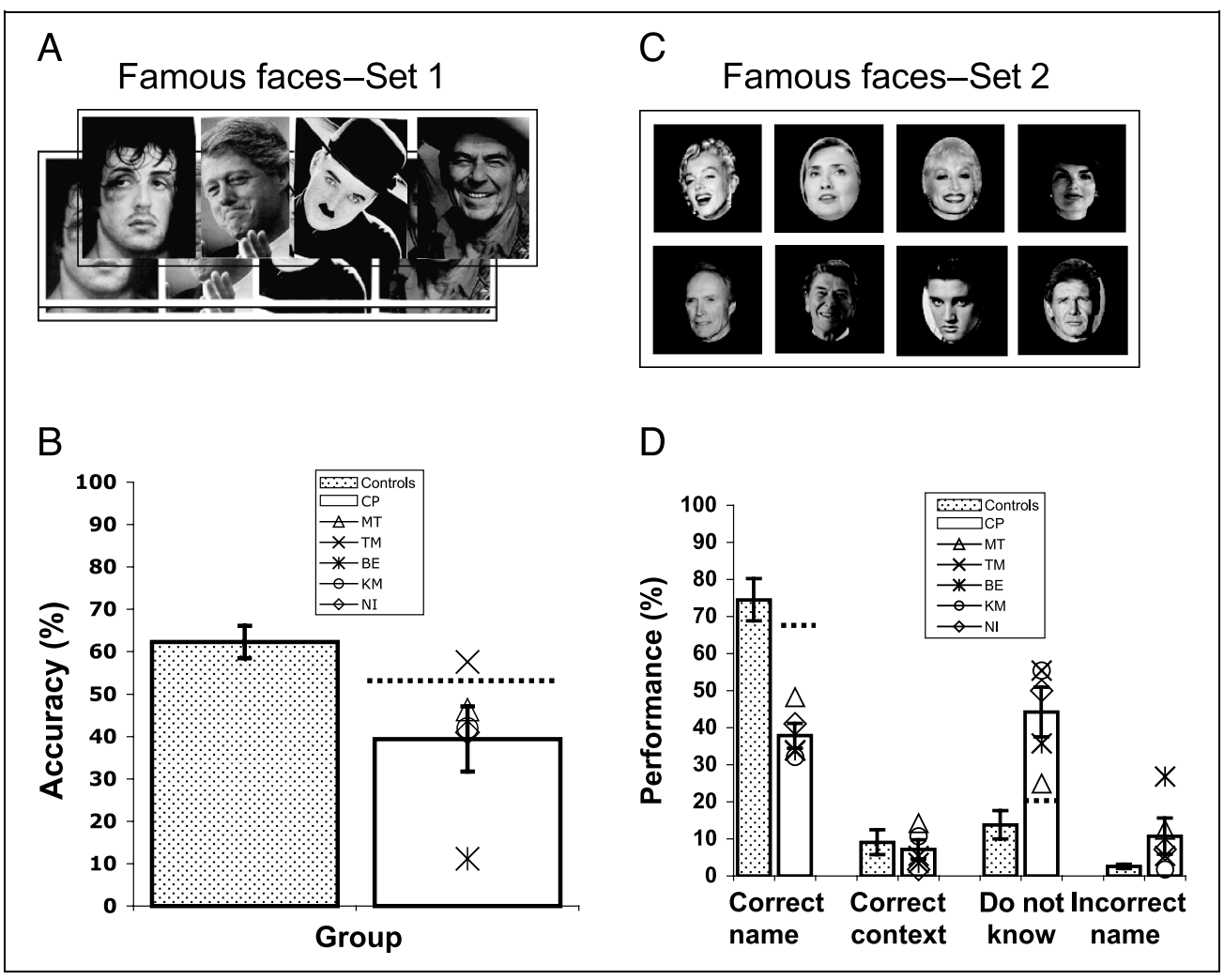


val. The possibility that $\mathrm{CP}$ subjects have had a lifetime to develop compensatory strategies and exploit any salient or diagnostic cues may explain subject TM's performance-for example, Ronald Reagan is shown in a cowboy hat and Sylvester Stallone is shown with a black eye (see Figure 1A). We therefore gathered a second set of 56 photographs of famous individuals, none of which contained any diagnostic cues (see Figure 1C), and an equal number of unfamiliar faces. Possible responses included the name of the individual, some contextual information (e.g., actor), "don't know", or an incorrect name. There was a significant interaction of Group $\times$ Response $[F(3,42)=15.5, p<.001]$, with the $\mathrm{CP}$ group identifying significantly fewer faces correctly, and making significantly more "don't know" and error responses than the controls (see Figure 1D). All five CP individuals fell outside the normal 95\% confidence intervals on the first two responses. The increased "incorrect name" responses in CP suggests that they are familiar with popular culture and celebrities and know the names but cannot assign them to the correct face. The main effects of group and condition were also significant $(F>1)$. These findings confirm the face recognition impairment in all $\mathrm{CP}$ individuals, especially when nonface cues cannot be exploited.

The next set of experiments examine whether the impairment is limited to the recognition of familiar faces, perhaps implicating a memorial or long-term representation component, or whether performance would also be impaired in discriminating unfamiliar faces, suggesting a more perceptual basis for the deficit. To assess this, all three groups completed a same/different discrimination task on a pair of unfamiliar faces (see Figure 2A). The CP subjects were significantly less accurate (85\%) than the control subjects (96\%), although both performed better than the AP subjects $(70 \%)[F(2,17)=$ $5.6, p=.01]$. Note, however, that the accuracy difference between CP and AP may result from a speedaccuracy tradeoff: The mean RT of the CP group (5285 msec) was significantly longer than that of the AP group ( $4235 \mathrm{msec}$ ), with both groups slowed relative to the control group $(1528 \mathrm{msec})[F(2,17)=7.8, p<$ .01]. This group effect did not interact significantly with condition (accuracy and RT; $F<1$ ), although all $\mathrm{CP}$ subjects performed more poorly numerically on individual than on gender judgments (see Figure 2B). Of the CP group, only TM, perhaps the most mildly affected $\mathrm{CP}$ individual (see Face Recognition above), fell within the $95 \%$ confidence intervals of the control group.

The groups also differ on $A^{\prime}$, with the CP (.88) and AP (.85) groups showing reduced sensitivity, relative to the controls $(.98)[F(2,17)=4.1, p<.05]$. We also note that the CP subjects were significantly slower than the controls on "same" trials with two identical images (4679 msec vs. $1660.8 \mathrm{msec})[t(1,303)=16.1, p<$ .0001], suggesting that the CP subjects are not just impaired at finding small discrepancies between the
A

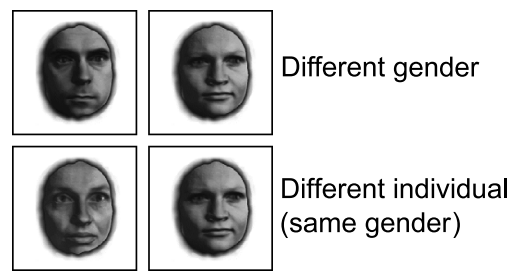

B

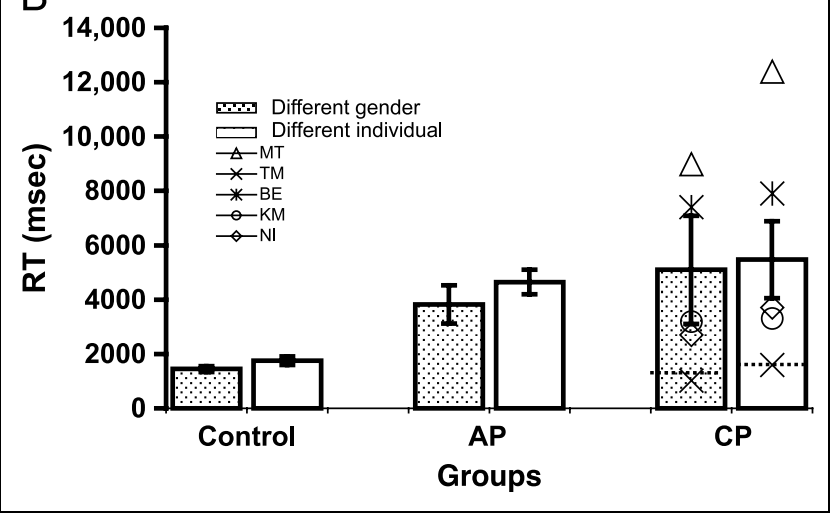

Figure 2. Face discrimination experiment. (A) Examples of stimuli for face discrimination experiment, including one trial where faces differ on the basis of gender and one trial where faces differ on the basis of individual identity. (B) Mean RT (and $1 S E$ ) for controls, AP individuals, and CP individuals (with individual symbols) as a function of gender $(G)$ and individual (I) discrimination. The dashed horizontal lines indicate the upper 95\% confidence interval calculated for the control group.

images and verifying them, but are also unable to appreciate the similarities between faces. These results indicate that the $\mathrm{CP}$ individuals are disproportionately impaired in unfamiliar face discrimination, relative to the controls, and show roughly the same degree of impairment as individuals with $\mathrm{AP}$, although there is some variability in the CP group. That the deficit is apparent in accuracy and in $A^{\prime}$ as well as in RT attests to the severity of the face impairment in CP especially because the stimuli are presented for an unlimited exposure duration. We have also replicated the deficit in matching faces in these same CP subjects using an $n$-back task (equivalent to sequential discrimination) in which subjects respond to a repeated face in a stream of sequentially presented faces (and withhold response if the faces differ). The CP group performs significantly more poorly than the control subjects and the group and individual data are shown in Figure 1B of the companion paper (Avidan, Hasson, Malach, \& Behrmann, 2005).

In the final face task, we confirmed the decrement in $\mathrm{CP}$ using a two-alternative forced-choice matching task with upright and inverted faces (see Figure 3A). The benefit in processing upright over inverted faces is one of the signatures of normal performance. Here, a target face appeared either upright or inverted and 
Upright and inverted face discrimination

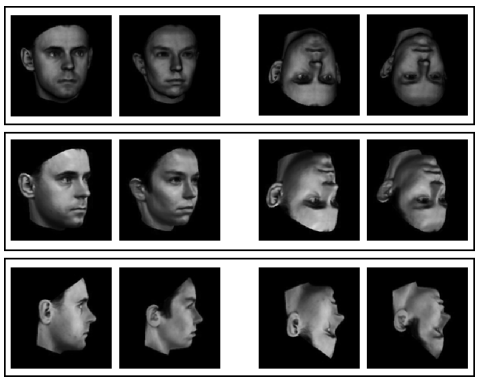

B

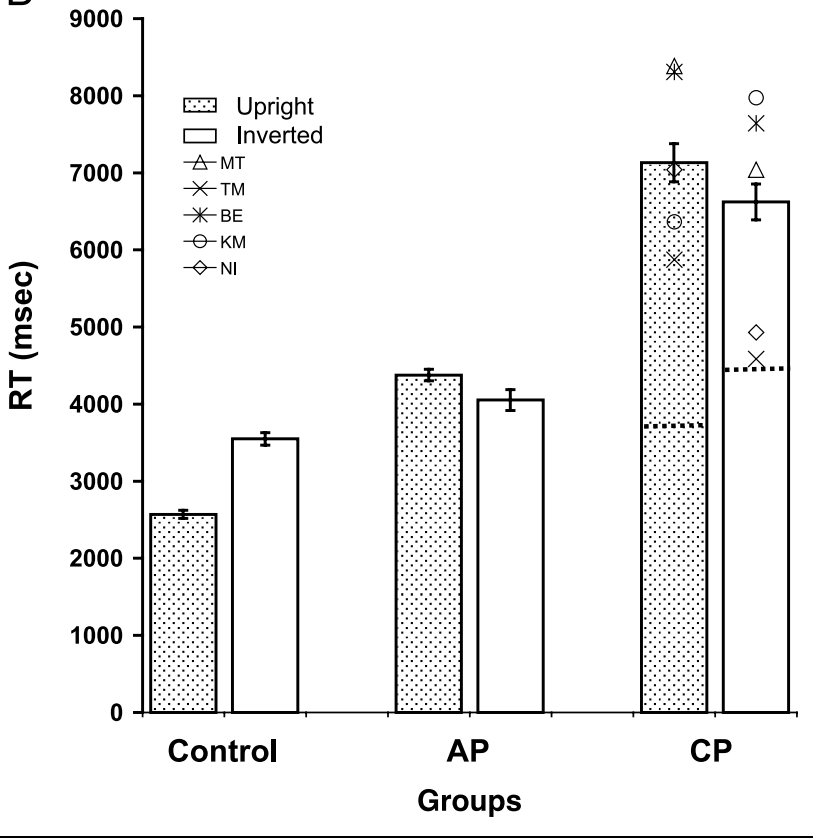

Figure 3. Face orientation discrimination experiment. (A) Examples of stimuli for two-alternative forced-choice face task, including upright and inverted stimuli presented in the frontal, three-quarters, and profile vantage points. (B) Mean RT (and $1 S E$ ) for controls, $\mathrm{AP}$ individuals, and $\mathrm{CP}$ individuals (with individual symbols) for upright and inverted faces. The dashed horizontal lines indicate the upper 95\% confidence interval calculated for the control group.

shown from the frontal, three-quarter, or profile view. Two choices appeared to the left and right, and subjects matched the target and choice. The task was designed to probe the impact of different orientations and different vantage points of the face, two factors well understood in normal face processing, on the CP's performance.

The CP (6877 msec) group responded more slowly than the AP (4215 msec) group, which, in turn, responded more slowly than the control group $(3060 \mathrm{msec})$ $[F(2,284)=65.7, p<.0001$; pairwise $p<.05]$. Interestingly, this differential slowing was qualified by the orientation of the stimuli $[F(2,284)=8.7, p<.001]$ (see Figure $3 \mathrm{~B}$ ): whereas the control subjects showed a 1011 msec advantage for upright over inverted trials, reflecting the typical upright superiority, the $\mathrm{CP}$ and AP individuals show a significant $510.5(p<.01)$ and $324 \mathrm{msec}(p=.05)$ advantage for inverted over upright faces, respectively. Of the CP individuals, only KM does not show this inversion superiority (see Figure 3B). Note that TM, whose face deficit is mildest on the other tasks, is remarkably impaired (perhaps because there are no obvious usable cues in the images). All five $\mathrm{CP}$ subjects fell out of the normal 95\% confidence interval for both upright and inverted faces. Accuracy on this task was significantly better for the controls (88\%) and CP (88\%) than for the AP (72\%) group. Importantly, the interaction of Group $\times$ Orientation is also present $[F(2,17)=5.9, p<.01]$ : The controls showed $9 \%$ greater accuracy for upright over inverted faces, the CPs as a group showed no difference for the two orientations and the AP showed a 7\% advantage for the inverted faces. Individually, four of the five CP subjects show no accuracy difference between upright and inverted trials and $\mathrm{BE}$ shows an inversion advantage $(8 \%)$. The absence of a clear upright advantage has been reported previously in cases with AP (Marotta, McKeeff, \& Behrmann, 2002; de Gelder, Bachoud-Levi, \& Degos, 1998; Farah, Wilson, Drain, \& Tanaka, 1998) and has been taken as evidence for the dissolution of configural face processing, an issue to be revisited in detail below.

In this same experiment, we also examined whether $\mathrm{CP}$ individuals retain the normal preference for threequarter over frontal or profile views of faces. The threequarter view affords an advantage in face learning and recognition (Moses, Ullmann, \& Edelman, 1996; Bruce, Valentine, \& Baddeley, 1987), contains the most useful features for recovering the identity of the face (Hill, Schyns, \& Akamatsu, 1997), is most often used to portray faces in pictures and is the best representation of the three-dimensional shape of the face (O'Toole, Edelman, \& Bulthoff, 1998). Although the control subjects show a 81-msec superiority for the three-quarter view of the target face over the frontal view which is $158 \mathrm{msec}$ faster than profile, this is not true for the AP or CP groups [Group $\times$ View: $F(4,568)=21.6, p<.0001$ ], who show no significant difference between threequarter and profile views. The same interaction of Vantage point $\times$ Group holds for the vantage point of the two choice faces $[F(4,568)=13.4, p<.0001]$ : In this case, all groups show the advantage for the threequarter viewpoint, but to a lesser extent in $\mathrm{CP}$ and $\mathrm{AP}$ than in the control group. No accuracy effects are present $(F<1)$. These findings are compatible with previous data showing that, in contrast with normal individuals, APs perform poorly in matching faces across viewpoint (Marotta, McKeeff, et al., 2002), especially for three-quarter view faces (Sergent \& Poncet, 1990). Of greater relevance, these findings replicate the face discrimination deficit in the $\mathrm{CP}$ individuals and are also 
consistent with studies showing poor matching across viewpoint (Nunn et al., 2001; Duchaine, 2000).

Taken together, the results of the three tasks of face processing (recognition, discrimination, orientation) indicate that all five $\mathrm{CP}$ individuals are impaired relative to the control group, although some CP individuals are more severely affected than others. On all tasks, four of the CP subjects fall outside of the normal 95\% confidence intervals. TM, the mildest subject, shows some variability but falls outside the confidence intervals on famous face recognition when no cues are present and also on upright and inverted face discrimination. Interestingly, the performance of the CP group is very similar to that of the AP group along a host of measures (no upright face advantage, no three-quarter view preference) and on different dependent measures (RT, accuracy, $A^{\prime}$ ), further attesting to the severity of their behavioral disorder. Interestingly, although the CP group appears to have adopted a strategy in which they make fewer errors but take a long time to respond, the AP group responds more quickly while making more errors.

\section{Perception of Nonface Objects}

An issue that continues to evade resolution is whether individuals with prosopagnosia, either acquired or congenital, have a deficit that is restricted to faces. To explore this, we used two nonface discrimination tasks, both employed previously to examine the specificity of the behavioral deficit in AP (Gauthier et al., 1999). In both tasks, a pair of stimuli was presented for same/ different judgment and the level of perceptual complexity of the judgment was manipulated.

The first experiment used common objects: On each trial, two stimuli appeared, either identical or differing at the basic (e.g., bird and chair), subordinate (e.g., two different types of bird), or exemplar level (e.g., two different instances of the same type of bird) (see Figure 4A). The $\mathrm{CP}$ and control groups were both more accurate (99\% and 98.3\%) $[F(2,17)=9.4, p<.001]$, and showed greater sensitivity $[F(2,17)=12.2, p<.0001]$, than the AP group ( $92 \%$ accurate) and there were no interactions with condition. In RT, main effects of group (AP slowest then $\mathrm{CP}$ then controls) $[F(2,17)=11.3, p<.001]$, and of condition $[F(2,17)=66.9, p<.0001]$ were observed as well as a significant interaction between them $[F(4,34)=10.4, p<.0001]$. The interaction reveals slowing in all groups as the discrimination became increasingly fine-grained, but to a greater extent in AP, than in $\mathrm{CP}$ and than in the controls (see Figure 4B). Note the variability among the $\mathrm{CP}$ subjects: At the basic level, only KM falls outside the upper 95\% confidence interval. At the subordinate level, all except NI fall outside the 95\% confidence interval and, at the exemplar level, KM, NI, and MT fall outside the confidence interval.

The second nonface experiment involved the discrimination of Greebles, novel 3-D-rendered objects

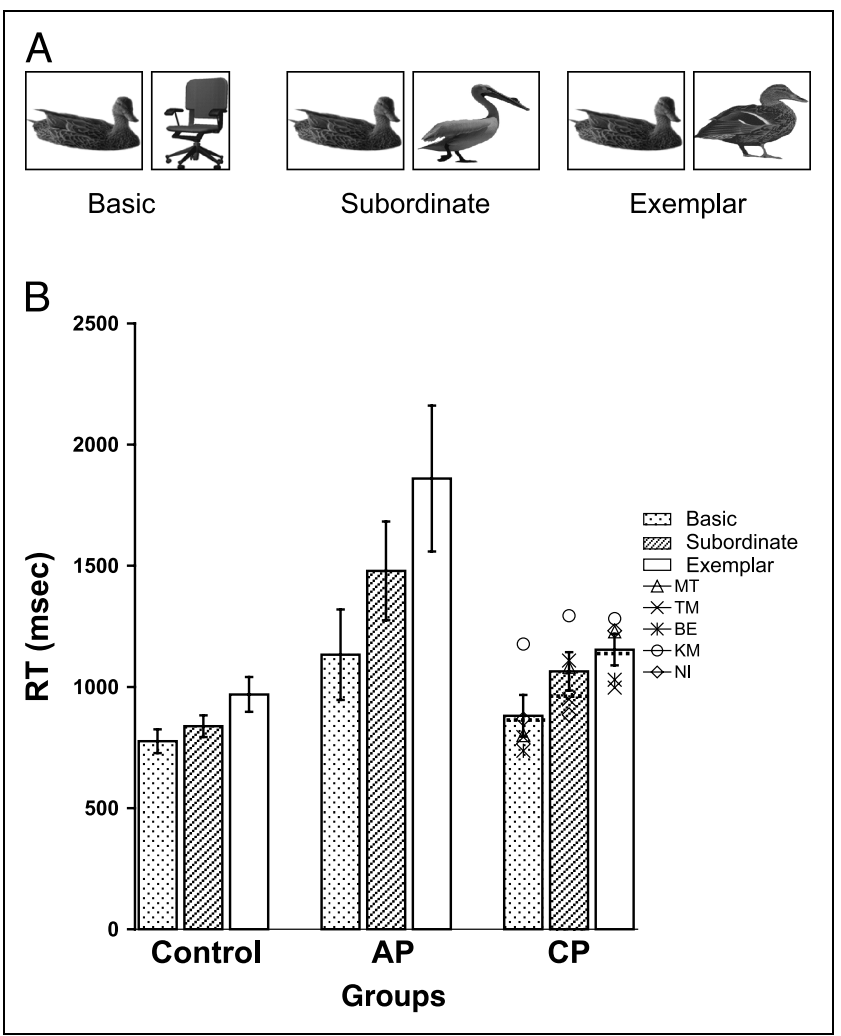

Figure 4. Examples of stimuli from object experiment. (A) Examples of stimuli for common object discrimination task, showing a pair that differs at the basic, subordinate, or exemplar levels. (B) Mean RT (and $1 S E$ ) for controls, AP individuals, and CP individuals (with individual symbols) for basic, subordinate, and exemplar levels of categorization. The dashed horizontal lines indicate the upper 95\% confidence interval calculated for the control group.

sharing the same basic elemental features in a canonical configuration with other members of the class (see Figure 5A). The set of Greebles is organized orthogonally along two categorical dimensions, with each Greeble being a member of a "family," defined by the central body shape, and of a "gender," defined by the orientation of the parts, upward or downward, relative to the central part. Although the parts are very similar to each other, every individual part is unique. Greebles are considered to be a good control stimulus for faces because, as for faces, local shape and surface features may not suffice for the purpose of discrimination of individual Greebles, given their perceptual similarity (Gauthier, Behrmann, \& Tarr, 2004). To differentiate individual exemplars of faces or Greebles, additional details and "configural" or relational information may be necessary (Gauthier \& Tarr, 2002; Maurer et al., 2002). In this task, two stimuli appeared on a screen, either the same or differing at the basic (e.g., a Greeble and a chair), family (e.g., main body part shape differed), gender (e.g., appendages orientation differs), or individual (e.g., same family and gender, different individuals) level (see Figure 5A). 
Figure 5. Examples of stimuli from Greeble experiment.

(A) Examples of stimuli from Greeble task, showing a pair that differs at the basic, family, gender, and individual levels. (B) Mean RT (and $1 S E$ ) for controls, AP individuals, and $\mathrm{CP}$ individuals (with individual symbols) for basic, gender, family, and individual level discrimination. The dashed horizontal lines indicate the upper $95 \%$ confidence interval calculated for the control group.

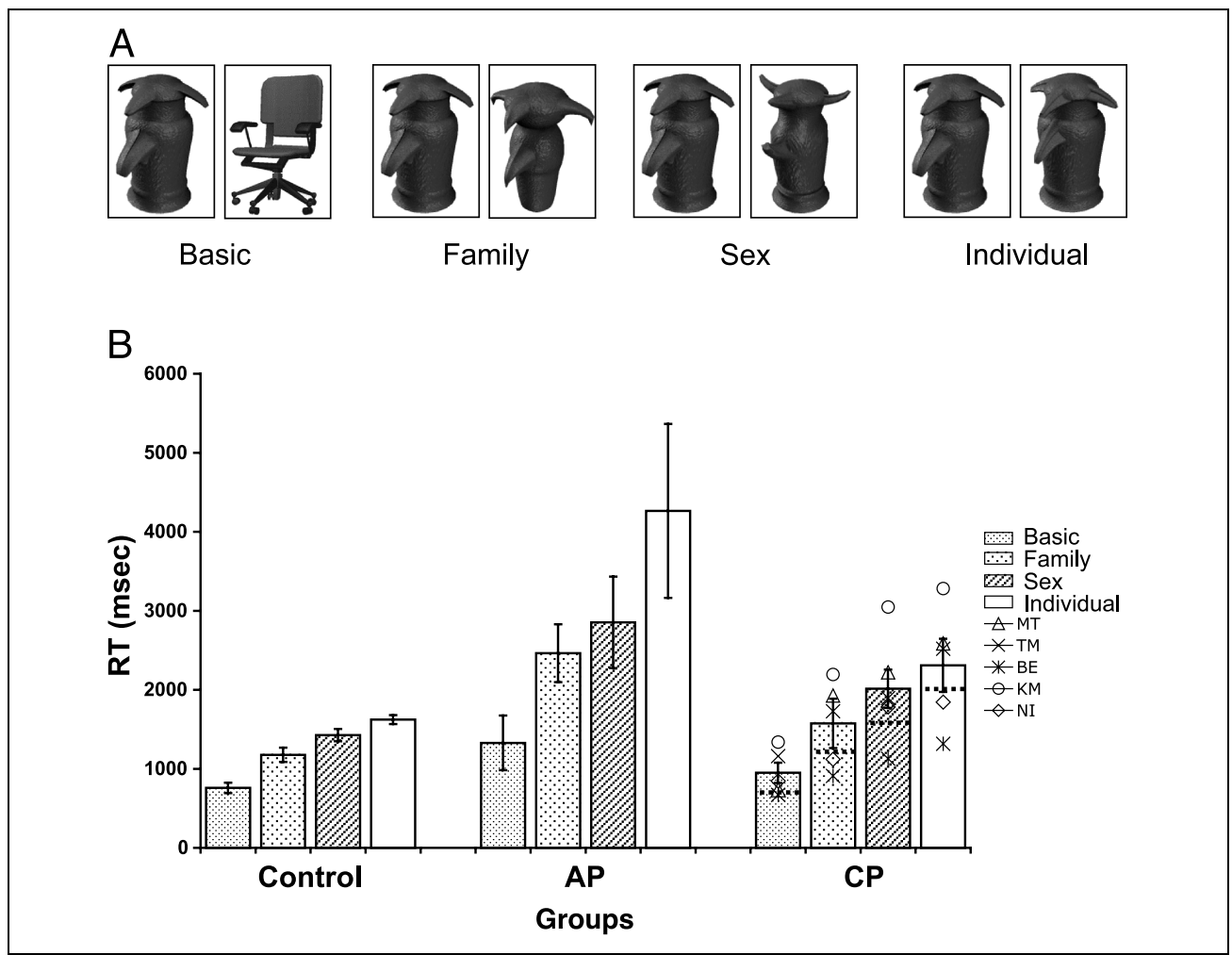

The control and CP groups were $98.6 \%$ and $97 \%$ accurate, compared with the AP group (95\%) $[F(2,17)=$ $3.6, p<.05]$, but this did not differ across condition $(F<1)$. The AP group was also less sensitive than the other two groups $[F(2,17)=4.7, p<.05]$, especially for the more taxing conditions, and the $\mathrm{CP}$ group's sensitivity also fell for individual-level discriminations [post hoc tests at $p<.05$; Group $\times$ Condition: $F(6,51)=2.8, p<.05]$. In RT, a significant main effect of group $[F(2,17)=13.6, p<.001]$, and of condition $[F(3,6)=41.7, p<.0001]$, were noted as well as an interaction between them $[F(6,51)=5.8, p=.0001]$. Although all groups showed the same rank ordering of conditions from basic to family, than gender and individual discriminations, the RT increase was exaggerated in the $\mathrm{CP}$ and $\mathrm{AP}$ groups, relative to the controls (see Figure $5 \mathrm{~B}$ ). Note again that there is some variability among the CP individuals: $\mathrm{KM}$ and TM always perform outside the normal 95\% confidence intervals as does MT (and sometimes NI), as the level of discrimination becomes more challenging. BE performs within the confidence intervals.

Taken together, the CP individuals, as well as the AP individuals, are impaired relative to the controls on both common object and novel object discrimination. All CP individuals show some level of impairment in processing nonface objects, although there is some variability among the individual $\mathrm{CP}$ subjects. For example, BE performs well (within 95\% prediction interval) on Greebles, but not always on objects, whereas TM performs poorly on Greebles and mostly within normal limits on objects. The impairment on nonface objects is clearly not as severe as for faces (compare Figures $2 \mathrm{~B}, 4 \mathrm{~B}$, and $5 \mathrm{~B}$ ) and is elicited primarily in RT rather than in accuracy or sensitivity. Importantly, the RT differences for objects and Greebles cannot be attributed to a generalized slowing as the CP subjects responded as quickly as the controls in the basic-level discriminations. Instead, the deficit becomes apparent for more fine-grained discriminations between perceptually similar stimuli. These findings suggest that the deficit extends beyond the processing of faces in $\mathrm{CP}$, and implicates a more widespread deficit but with exaggerated consequences for face processing.

\section{Configural Processing}

The absence of a benefit for upright over inverted faces in the $\mathrm{CP}$ individuals already provides an indication that configural processing is affected in CP. To evaluate the extent of the deficit in tracking the spatial relations between local components in $\mathrm{CP}$, we conducted two additional experiments. Importantly, neither experiment uses faces as stimuli, allowing us to examine the integrity of the relational processing independent of faces. Both experiments have been shown to be sensitive to failures in configural processing in patients with visual integrative agnosia (Behrmann \& Kimchi, 2003) and in autistic individuals known to have a bias to local elements rather than to global shape (Behrmann, Avidan, et al., 2005). 
The first experiment uses compound or hierarchical letters in which the identity of the local letters is either consistent or inconsistent with that of the global letter (see Figure 6A). In separate blocks of trials, subjects identify the letter, via button press, at either the local or the global level. Global identification requires interactions between constituents, necessitating configural or holistic processing (Navon, 2003). All else being equal, normal subjects identify the global faster than the local letter, and conflicting information between them exerts asymmetrical global-to-local interference (Navon, 1977).

The critical result (see Figure $6 \mathrm{~B}$ ) is a three-way interaction between Group $\times$ Consistency $\times$ Globality $[F(1,1672)=15.6, p<.0001]$. The control subjects responded quickly and showed no statistical difference between global $(537 \mathrm{msec})$ or local $(526 \mathrm{msec})$ identification (possibly because of the salience and sparsity of the local elements in the display): There is a slight

\section{A}

Compound stimuli

\begin{tabular}{|c|c|}
\hline \multicolumn{2}{|c|}{ CONSISTENT } \\
\hline $\begin{array}{l}\mathrm{sss}_{\mathrm{s}} \\
\mathrm{s} \\
\mathrm{s} s \mathrm{~s} \\
\mathrm{~s} \\
\mathrm{sss}\end{array}$ & $\begin{array}{l}\mathrm{H} \\
\mathrm{H} \\
\mathrm{H} H \mathrm{H} \\
\mathrm{H}\end{array}$ \\
\hline \multicolumn{2}{|c|}{ INCONSISTENT } \\
\hline${ }_{H}^{H_{H}}$ & $\begin{array}{l}\text { S } \\
\text { s } \\
\text { S S s } \\
\text { s }\end{array}$ \\
\hline
\end{tabular}

B
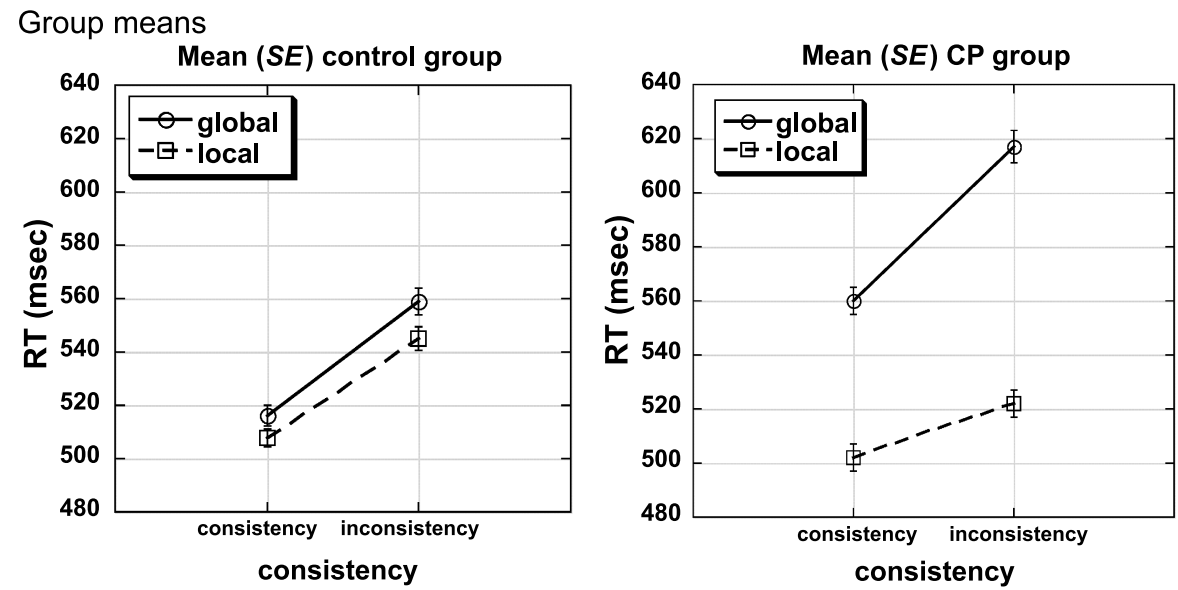

C

Plots for individual CP subjects

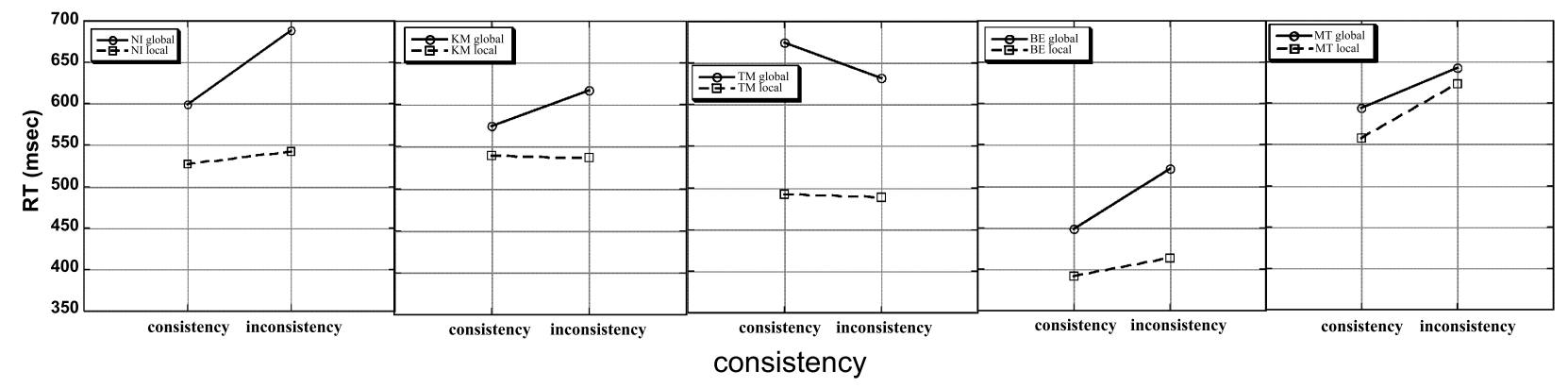

Figure 6. Examples of stimuli and results of global/local task. (A) Four compound stimuli, two of which are consistent and share identity at the global and local level and two of which do not share identity at the global and local level. (B) RT (and $1 S E$ ) for means for control group and CP group for global and local identification as a function of consistency. (C) Mean RT for each CP individual for global and local identification as a function of consistency. 
advantage for global in seven and a slight advantage for local identification in the remaining five individuals. Five controls show an interaction in their data with, to varying extents, greater interference from global to local in the inconsistent condition. For the most part, however, the variability among the control subjects is rather small and their performance provides a robust benchmark against which to compare the $\mathrm{CP}$ performance.

The CP group performs more slowly than the controls $[F(1,1672)=7.9, p<.005]$, especially for global identification $[F(1,1672)=344.9, p<.0001]$, attesting to the difficulty in deriving a global entity from local elements. This slowing is, however, further exaggerated when the local letters are inconsistent with the global identity, reflecting asymmetric local-to-global interference $[F(1,1672)=29.4, p<.0001]$. This asymmetry indicates the precedence for the local elements in $\mathrm{CP}$ and reflects the cost associated with the effort to derive the global shape. As is evident from Figure $6 \mathrm{C}$, all five $\mathrm{CP}$ individuals show the local precedence (marginally significant in MT). Three show the asymmetric local-to-global interference, MT shows no real effect (perhaps because of a ceiling effect), and TM, somewhat inexplicably, shows slightly greater interference in the consistent than inconsistent case. There are no significant group effects on accuracy, although effects of consistency $[F(1,16)=5.1$, $p<.02]$ and globality $[F(1,16)=5.2, p<.05]$, and the interaction between them $[F(1,16)=6.3, p<.05]$, are all present.

To evaluate the relationship between performance on this task and on the face discrimination task, we correlated the local-to-global interference in RT [inconsistent (global - local) - consistent (global - local)] and the average RT in face discrimination. An $r^{2}$ of .71 was obtained ( $p=.07)$, which, although marginal, is highly suggestive in only five subjects, and is indicative of a positive relationship between face processing and deriving a global configuration.

This final experiment was designed to probe further the integrative abilities of $\mathrm{CP}$ subjects and to focus more specifically on the processes, whereby the spatial relations of the local elements are configured into a global whole. In this microgenetic approach, the time course of the development of the global percept is examined, rather than just the final outcome of these processes as reflected in a single RT value. The primed matching paradigm (Beller, 1971) used here involves presentation of a priming stimulus followed immediately by two probe test figures. Participants judge, as rapidly and accurately as possible, whether the two test figures are the same or different and ignore the prime. The speed of same responses to the test figures depends on the representational similarity between the prime and the test figures: Responses are faster when the test figures are similar to the prime than when they are dissimilar to it. By varying the duration of the prime, we can tap earlier and later internal representations (Behrmann \& Kimchi, 2003;
Kimchi, 1998, 2000; Sekuler \& Palmer, 1992), enabling us to assess implicitly the participant's perceptual representations and the time course of their organization.

Two types of test pairs are used, defined by their prime-test similarity (see Figure 7A): In the ElementSimilarity (ES) test pairs, the test figures are similar to the prime (e.g., global diamond made of circles) in their local elements (circles) but differ in global configuration (square), and in the Configuration-Similarity (CS) test pairs, the figures are similar to the prime in global configuration (is a global diamond) but differ in local elements (squares). Of additional interest is the number of elements in the display: The prime and probe patterns consist of a few or of many local elements (see Figure 7A). Displays with few elements tend to bias in favor of local processing, whereas those with many elements favor global processing (Bacon \& Egeth, 1991).

The experiment consisted of the factorial combination of four factors: prime type (few-element or many-element); prime duration (40, 90, 190, 390, or $690 \mathrm{msec}$ ); test type (ES, CS); and response ("same" or "different"). Typically, normal participants show that the availability of elements and configuration depends on the number and relative size of the elements (Kimchi, 1998): Few, relatively large elements are available early on in processing (ES advantage) and they are grouped into a global configuration with time. For many-element patterns, the configuration is available very early (CS advantage), followed by the individuation of the elements. The question is whether the global configuration is available to the $\mathrm{CP}$ individuals, and if so, how early in time.

As can be seen in Figure 7B, the data for the $\mathrm{CP}$ and control groups appear rather different $[F(1,134)=4.9$, $p<.05]$. For the control subjects, for the few element displays, there is an advantage for test pairs sharing the elements (ES) with the prime over those sharing the configuration (CS), and this holds roughly across all prime durations. For the many-element displays, at earlier prime durations, there is a reduction in RT, relative to the few-item displays, but only for the test pairs that share configuration (CS). Note also that at $40 \mathrm{msec}$, the earliest duration used, there is a suggestion of an increase in the ES trials, potentially as a result of a competitive effect from dissimilarity in configuration. At longer exposure durations, there is again an advantage for the ES trials. The major finding is that, at early durations, the presence of many elements facilitates the derivation of a configuration and produces fast responses for CS over ES trials, relative to the few-element trials. Critically, the global configuration is derived early and rapidly when many elements are present.

The CP group diverges from the normal pattern in two major ways. The CP group also shows the advantage for ES over CS few-element trials, except to a marginally $(14 \mathrm{msec})$ greater extent, suggestive of an exaggerated 
Figure 7. Examples of stimuli and results of few/many

microgenetic experiment.

(A) Primed match paradigm: probes, consisting of few and many elements, are followed, after varying SOAs, by test pairs which require "same" or "different" responses and which are similar to the prime in elements or configuration.

(B) Group means of RT (and $1 S E$ ) for controls and $\mathrm{CP}$ individuals for few and many displays, shown for same-configuration (CS) and same-element (ES) trials as a function of SOA.
A

Microgenesis stimuli

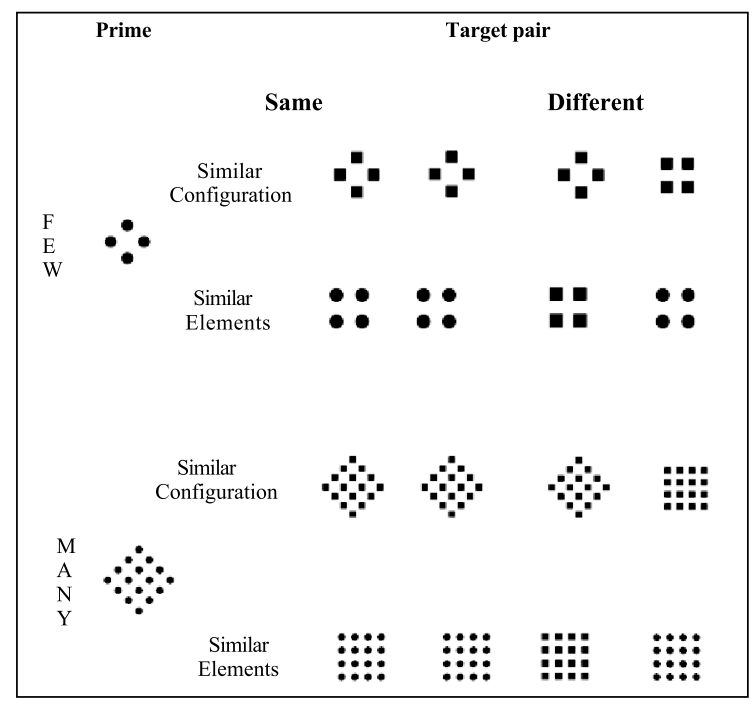

B

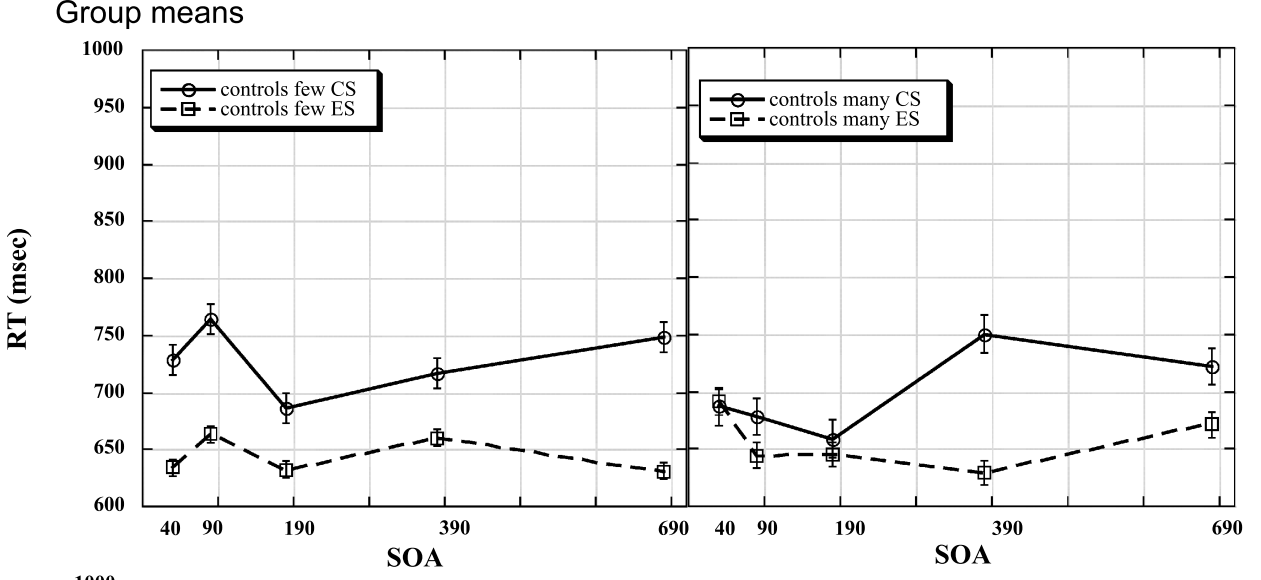

representation of the local elements in $\mathrm{CP}$. The other, and perhaps more striking, difference is the absence of the CS over ES benefit of many elements at any duration. Instead, RTs are longer for the CS than ES pairs and both of these are longer than for the few-item pairs across all exposure durations, reflecting the cost associated with the presence of more local elements. In sum, there is no obvious facilitation for CS test pairs at any exposure duration even for the many-element trials. Rather, there is a clear ES advantage at all exposure durations, attesting to the difficulty in integrating the local elements in CP.

These patterns are supported statistically. The CP subjects are slower than the controls $[F(1,134)=5.9$, 
$p<.01]$, especially for the many-element displays $[F(1,134)=25.6, p<.0001]$. There is also a difference as a function of duration with control subjects but not with CP subjects, showing a RT reduction for CS trials $[F(4,536)=8.5, p<.0001]$, especially for the many-item CS displays $[F(1,134)=2.8, p<.05]$. There are no statistically significant effects involving group in the accuracy data $(F<1)$, but this may not be surprising given the simplicity of the task.

In summary, for the few-element patterns, independent of prime duration, both groups showed an ES advantage, somewhat exaggerated in the $\mathrm{CP}$ individuals, indicating the precedence for the local elements. For the many-element patterns, the group difference is even clearer: For normal participants, many relatively small elements are rapidly grouped without the individuation of the elements that occurs later in time (Kimchi, 1998). Such early grouping is functional for a system whose goal is object identification and recognition, because many small elements in close proximity to one another are more likely to be texture elements of a single object rather than discrete objects. No rapid grouping of the many elements was observed for the $\mathrm{CP}$ individuals and, instead, we see an advantage for ES trials, to an even greater extent than for the fewelement condition. A comparison of the CP individuals to the controls reveals that four of them show the very pattern described here. TM, the least affected CP subject, exhibits an ES advantage in the normal range for the few patterns and an advantage for CS for the many-element display. The only other divergence from the group pattern is that $\mathrm{BE}$ shows a marginal, but nonsignificant, trend towards a CS advantage for the many-element trials.

We note the compatibility of these findings with those from the global/local experiment, especially for the many-element displays. Normal subjects evince configural processing in both tasks. In contrast, in neither task do the $\mathrm{CP}$ individuals exhibit the ability to take into account relations between the local elements to derive a global configuration. Importantly, the failure to perceptually integrate the information appears to be related to the deficit in face processing. We note that the difference between CP and controls in these tasks is roughly $100 \mathrm{msec}$ or so, far smaller than the discrepancy in face processing. The stimuli used in these configural experiments are very dissimilar to faces and are perhaps the simplest configural stimuli one might construct, and yet a group difference is still obtained. Given that the CP subjects showed a deficit even with these images suggests that the configural deficit is striking. Presumably, if one were to manipulate the spatial relations versus features of faces, as some have done in order to elicit a configural deficit (Le Grand, Mondloch, Maurer, \& Brent, 2001; Freire, Lee, \& Symons, 2000), the group differences would be easily uncovered and would be magnified substantially.

\section{Low-level Visual Processing}

Before concluding that $\mathrm{CP}$ individuals are impaired at configural processing, we need to rule out two possible alternative explanations. The first possibility pertains to a differential sensitivity to high and low spatial frequencies. Several researchers have suggested an involvement of spatial filters, based on spatial frequency channels operating at early visual processing (Ginsburg, 1986), in the perception of global and local structures. No latency advantage for global over local processing is found when low spatial frequencies are removed from hierarchical stimuli (Lamb \& Yund, 1993; Badcock, Whitworth, Badcock, \& Lovegrove, 1990; Hughes, Fendrich, \& Reuter-Lorenz, 1990; Shulman \& Wilson, 1987; Shulman, Sullivan, Gish, \& Sakoda, 1986), suggesting that the global advantage effect is mediated by low spatial frequency channels. The CPs' differential inability to perceive the global form of a hierarchical stimulus might then arise from a limitation in processing low spatial frequency information. To rule this out, we

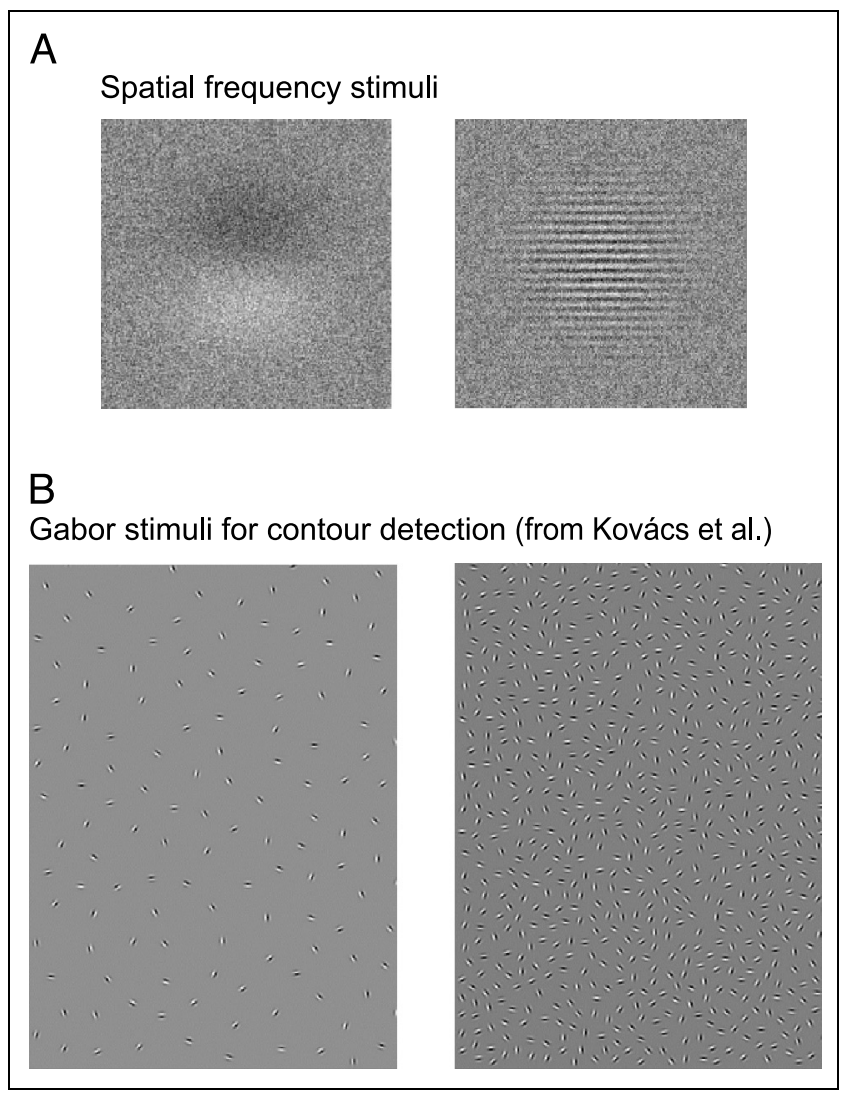

Figure 8. Examples of stimuli for testing low-level vision. (A) Examples of stimuli used to establish contrast thresholds across different spatial frequencies. Examples here are 1 cycle per inch (left) and 30 cycles per inch (right). In a sequential paired task, subjects indicate whether the first or second stimulus in the pair contains the grating. (B) Examples of stimuli used to test contour detection ability (from Kovács et al., 1999). Stimuli vary as a function of the number of Gabor patches in the image and the distance between them. Subjects indicate on each card the location of the collinear contour. 
obtained log contrast spatial frequency thresholds across the frequency range $(0.3,1,3,10,30$ cycles per inch; see Figure 8A). All CP individuals performed within $1 S D$ of the mean of the normal control subjects and fall within the 95\% prediction interval (see Table 1).

The other alternative explanation is that the failure to take spatial relations between elements into account emerges not from a problem in deriving configurations but from a low-level problem establishing collinear relations, usually thought to depend on lateral connections in V1. To examine whether CP individuals could interpolate contours, we used a method that has been successful with various pathological populations (Kovács, Polat, Pennefather, Chandna, \& Norcia, 2000). Subjects are shown displays (see Figure 8B) of small Gabor patches, some of which are oriented in the same direction, and are asked whether they can discern a contour. The displays ascend in difficulty with increasing number of distractors and reduced distance between these and the contour. Whereas normal individuals obtain a mean threshold of 0.65 , the mean for the CP individuals is 0.61 , and all fall within the normal confidence interval (see Table 1). Any failure to derive configural relations in $\mathrm{CP}$ is, therefore, not simply the result of a low-level visual impairment and likely arises at a higher-level of the visual cortex.

\section{DISCUSSION}

The goal of this investigation was to address several outstanding questions about CP, a disorder in which individuals are disproportionately impaired at face processing in the absence of an underlying neural concomitant or cognitive deficit and in the absence of a low-level visual processing disorder. The specific questions pertain to the nature and severity of the face processing impairment, its specificity, and its relation to a more fundamental deficit in deriving configural information from visual images. The answers to these questions not only have relevance for this population, but can be used to inform theories of object recognition and the psychological and neural representation of faces versus other objects.

\section{Nature and Severity of Face Processing Impairment}

The data obtained across three experiments suggest that the CP individuals are impaired at assigning identity to faces and at discriminating between novel faces, especially as the discrimination becomes more taxing (differentiating gender vs. individual identity). Moreover, the representations derived by the $\mathrm{CP}$ individuals differ from those of normal individuals: Faces appear to be coded in a more featural or part-based fashion as reflected by the finding that performance is not adversely affected when faces are inverted, in contrast with the well-established decrement under inversion for normal individuals (Leder \& Bruce, 2000; Yin, 1969). The results also indicate that there is a range of severity across the $\mathrm{CP}$ individuals, although all are impaired to some extent and all acknowledge and are aware of the disorder in their daily lives. In fact, the personal anecdotes provided to us by these patients sound almost identical to the reports of individuals with AP. For example, BE reports that she has failed to recognize her mother on many occasions and MT is unable to identify his children at day care and is forced to rely on one of the caretakers to bring the children to him. KM did not recognize her son when he had a haircut nor did she recognize her husband when he changed his clothing prior to a ceremony they were attending.

A distinction that has often been made in the literature is between apperceptive versus associative prosopagnosia (De Renzi et al., 1991). Patients with the former, more perceptual form are impaired at face matching, whereas those with the latter, more memorial form are impaired at associating the percept with the long-term memory of the face. The $\mathrm{CP}$ patients described here are all impaired at face recognition, but are also all impaired at face matching, placing them in the apperceptive class. Although they may be able to make more basic facial judgments, such as determining sex, age, and expression (Nunn et al., 2001), they appear unable to derive a structural description of the face as evidenced by their poor face discrimination. The failure to encode the faces structurally precludes access to long-term memories (Bruce \& Young, 1986).

\section{Specificity of Face Processing Impairment}

Not only are the CP individuals impaired at face processing but, as is apparent from the experiments with common objects and with Greebles, they are also all affected to some extent in their recognition of nonface visual objects. Note, however, that their nonface processing is not as severely affected as their face processing and the deficit is largely evident in their slowed processing rather than in accuracy. Also, the deficit is not apparently a function of general perceptual slowing as the basic-level discriminations are performed well.

These findings are important in the context of the ongoing debate about the specificity of prosopagnosia, both in congenital and acquired cases. One of the most transparent and appealing interpretations that has been offered for AP is that it reflects damage to face-specific processes (Farah, Rabinowitz, et al., 2000; Farah, 1990), while leaving unaffected the recognition of other types of visual stimuli. Although there is still support for such a view, others argue that the deficit extends beyond faces and that a similar pattern of impairment would be evident on other objects if the demands of the face and nonface task were equated (Gauthier et al., 1999). This controversy has not been resolved and the reader 
is referred to recent articles for details (Grill-Spector, 2003; Grill-Spector, Knouf \& Kanwisher, 2004; Tarr \& Cheng, 2003; Maurer et al., 2002; Haxby et al., 2001; Kanwisher, 2000; Tarr \& Gauthier, 2000).

The generality of the deficit is also controversial in previous $\mathrm{CP}$ studies. For example, Subject $\mathrm{AB}$ is unable to name flowers or motor cars and exhibits poor withincategory object recognition (McConachie, 1976) and LG (Ariel \& Sadeh, 1996) is impaired at object recognition, assessed in a variety of ways. However, EP (Nunn et al., 2001) performed within normal limits on withincategory discriminations of flowers, cars, and famous buildings, and YT (Bentin, Deouell, et al., 1999), Dr S (Temple, 1992), and TA (Jones \& Tranel, 2001) are reported to perform normally on a range of visual stimuli (see also Duchaine \& Nakayama, 2004, using a memory test). Note that the methods of assessment vary widely across case reports and, in most instances, accuracy is the primary dependent measure. It is clear both from our findings and from other neuropsychological investigations that accuracy alone is inadequate as a dependent measure, as these subjects may trade off accuracy against speed or show a difference in bias or sensitivity compared with control subjects (Gauthier et al., 1999; Kosslyn, Hamilton, \& Bernstein, 1995). A clear example of the pitfall of measuring accuracy alone is evident from data from another CP individual, TA, who performed normally on object identification, based on 100 objects from the Snodgrass and Vanderwart set (Duchaine, Nieminen-von Wendt, New, \& Kulomaki, 2003). However, a more precise and refined evaluation of his performance using an old/new discrimination paradigm with horses, cars, tools, guns, and sunglasses revealed impaired performance in four of these five categories. Without detailed and systematic assessment of a range of dependent measures and the use of tasks that are as demanding in object recognition as in face recognition, however, the extent to which $\mathrm{CP}$ is truly face specific remains an open issue (for a similar discrepancy in RT and accuracy in visual object agnosia, see Delvenne et al., 2004; Gerlach, Marstrand, Habekost, \& Gade, in press). All of the CP individuals tested in the present study were affected to a greater or lesser degree on nonface visual object recognition, suggesting that the underlying impairment may be more widespread than has sometimes been assumed. Note, however, that all subjects performed more poorly on faces than on any other stimulus class.

\section{Relation of Face Processing Impairment and Configural Representation}

The idea that faces are processed in a configural fashion whereas nonface objects are processed in a more piecemeal or feature-based way has received widespread support. The absence of an inversion effect for faces in our CP group is suggestive of a deficit in deriving the relational or second-order statistics from faces. Other case studies report similar results in AP and CP (de Gelder \& Rouw, 2000). Although there remains some disagreement on what constitutes "configural" (Gauthier \& Tarr, 2002), most researchers agree that face inversion affects configural processing (Maurer et al., 2002; Leder \& Bruce, 2000) and the absence of the inversion decrement suggests an abnormal reliance on a featural mode of representation.

How this inversion effect emerges is an open question. One possibility is that configural processing is performed by a prespecified system, perhaps mediated by the fusiform gyrus. If these subjects' deficits were due to an inability to develop configural processing in general, then CP subjects should perform similarly with upright and inverted faces because configural processing would not have developed for either. However, most CP subjects are better at inverted than upright faces. It is possible, then, that through experience, in normal individuals, the benefit from repeated exposure to upright faces optimizes the derivation of face configurations (hence, the activation of the fusiform gyrus for faces; Barton et al., 2002). Given that this system is not functioning properly, only some rudimentary configural processing develops in CP (perhaps sufficing for objects). The configural processing is circumvented during processing inverted faces and the better-developed part- or local-based system can proceed apace with little interference from the configural system, giving rise to the inversion superiority effect (Barton, Zhao, \& Keenan, 2003).

To examine the link between the ability to derive spatial relations in the service of global shape description and face recognition, we conducted two further experiments, and to push the link between configural and face processing further, neither employs face stimuli. Rather, the stimuli are made of very simple shapes, which are substantially different from faces. Notwithstanding this difference, the results indicate that the CP subjects do not perform normally, showing greater bias than the control subjects towards the local components. The $\mathrm{CP}$ subjects are also disproportionately slow at integrating the elements into a global shape, especially when their identity conflicts with that of the global shape. On an even more fine-grained measure, which tracks the microgenesis of the global shape derivation, the CP individuals are not primed by a stimulus that shares the configuration with the displays to be judged nor are they able to benefit from the presence of many elements, which usually facilitates global processing especially at early durations after stimulus onset. Moreover, there is some support for a correlation between the enhanced local bias and the impairment in face discrimination.

Despite the apparent clarity of these findings, there is no universal consensus for a deficit in configural processing in $\mathrm{CP}$ and, again, this divergence might arise from the different methods used to test configural processing as well as the different conceptualization of 
configural processing. Some studies have used tests of Gestalt completion or two-tone Mooney faces to test CP individuals and the results are mixed: EP (Nunn et al., 2001) and AB (McConachie, 1976) were impaired, relative to their controls on Mooney faces and EP was also impaired at making discriminations with chimeric faces where matching on base of individuals features is inadequate and a global configuration is required for success at this task (Nunn et al., 2001). In contrast, Dr S (Temple, 1992) and TA (Jones \& Tranel, 2001) apparently performed within normal limits on the Mooney test (on accuracy). In a particularly pertinent recent study, Duchaine (2000) suggests that configural processing is unrelated to $\mathrm{CP}$. His patient, $\mathrm{BC}$, performed well on three tests of pattern completion, all of which required the assembly of black and white fragments, either with information missing or amidst noise, leading to the conclusion that $\mathrm{BC}$ has intact configural processing. The critical issue, however, is whether the processes involved in fragment completion are akin to those used to derive a global identity. Establishing spatial relations between components of a display or extracting a figure from the ground, may well depend on different processes used to interpolate between fragments (see low-level processing test above which shows intact performance in all CP subjects here) and group local elements (Palmer, 2003). Whether BC (and other CP individuals) would be impaired at deriving global shape using the paradigms employed here remains an open issue.

An additional question that remains to be addressed is exactly what constitutes "configural" processing and whether the configural processing required for faces (and for other objects) is the same as that required for global/local and few/many element processing. The definition of configural processing is highly controversial and is the focus of many investigations (Gauthier \& Tarr, 2002; Maurer et al., 2002; Leder \& Bruce, 2000; Moscovitch et al., 1997). In general, configural refers to the perception of relations among the features of a stimulus and at least three main types of configural processing can be delineated: (i) first-order relations among elements-for example, processing the presence of two eyes above a nose; (ii) second-order relationslocal elements are processed in a relational manner (e.g., nose-mouth distance); and (iii) holistic processingthe features or local elements are glued together into a gestalt. Although the distinctions are reasonable and clear, many outstanding issues remain such as the exent of the superadditivity of the features in the holistic case, the ability to access the local elements in the holistic case, and the relationship between these different forms. Understanding what constitutes configural processing, how the elements are represented in relation to each other, and whether the same form of configural processing applies across all visual stimuli is critical for future research.

\section{Relation of CP to Other Neuropsychological Deficits in Pattern Recognition}

The deficit in visual face processing is the signature of $\mathrm{CP}$ and, as we have shown, a lesser deficit in nonface recognition is also present. The link between $\mathrm{CP}$ and configural processing is also well supported. This evidence parallels the findings from individuals with acquired lesions who are also profoundly impaired at face processing and who also fail to derive global configurations on the same global/local and few/many elements used here. The term "integrative agnosia" is applied to these individuals to highlight the significant deficit in grouping together local elements (Behrmann \& Kimchi, 2003; Humphreys, 1999; Riddoch \& Humphreys, 1987). The argument has been made that, in these agnosic individuals, the failure to take the spatial relation into account would significantly impact face recognition, and perhaps, to a lesser extent, the recognition of other nonface objects. This assertion is mirrored in the data from the $\mathrm{CP}$ individuals presented here.

It appears that just as a deficit in visual pattern perception may arise congenitally and without an obvious neural basis, parallel disorders exist in both the visual (developmental dyslexia) and the auditory domain (congenital amusia). In both cases, the deficits (in reading and music perception) are not explained by sensory or brain anomalies, low intelligence, or deprivation (Demonet, Taylor, \& Chaix, 2004; Peretz \& Hyde, 2003). Indeed, similar questions concerning the specificity of the disorder to reading or to music have also been asked. For example, in the context of dyslexia, questions concerning rapid sequential processing, phonological coding, magnocellular involvement, or cerebellar impairment as the basis of the reading impairment are asked (Demonet et al., 2004). In the case of amusia, issues about pitch versus temporal coding and the specificity of the deficit to music versus other form of auditory input are under investigation (for review, see Peretz \& Hyde, 2003). Of interest too is that, like CP (see below), these neurodevelopmental disorders may be genetically determined (Peretz \& Hyde, 2003; Francks, MacPhie, \& Monaco, 2002).

\section{Familial Effects}

One of the interesting findings in our investigation of $\mathrm{CP}$ is that the deficit often appears to affect more than one member of a family. Of the nine individuals we originally tested, only five met our very conservative criteria for admission to this study but all showed some degree of impairment (see experimental methods). Within this sample, three families were represented: $\mathrm{KM}$ and TM are a mother and a son. BE's mother was tested but did not meet inclusion criteria and the three other excluded individuals are all siblings. MT's father is apparently CP but has not been tested yet. 
In almost all reports of $\mathrm{CP}$, there is mention of a familial connection, although this is only systematically evaluated in very few instances. For instance, Dr S reportedly has a first cousin who is also CP, and all the children of Dr S are color blind, also suggesting a hereditary linkage (Temple, 1992). In a different study, two daughters and the father in one family showed severe impairments in face recognition (De Haan, 1999), although the other family members were unaffected. The need for stringent testing, however, is critical as the brother in this family is a selfdeclared CP and yet was not objectively classifiable as such. The findings indicate a potential genetic basis for $\mathrm{CP}$ and one recent study suggests that the cumulation segregation ratios are compatible with a simple autosomal dominant mode of inheritance (Grueter, Grueter, Bell, Horst, Laskowski, Sperling, Halligan, Ellis \& Kennerknecht, in press). We are also starting to investigate the genetic foundation more systematically in the individuals we have tested to date.

\section{Underlying Neural Substrate}

An obvious question which arises concerns the extent to which the behavioral impairment can be traced to any possible alteration in neural substrate in CP. A few studies have addressed this issue and, again, the results are somewhat contradictory. Two ERP studies report a reduced difference in the N170 waveform in the three tested subjects (Kress \& Daum, 2003b; Bentin, Deouell, et al., 1999). One of the two fMRI studies reports normal BOLD activation for faces in their CP subject (Hasson, Avidan, Deouell, Bentin, \& Malach, 2003), but the other reports no activation for faces, relative to other objects (Hadjikhani \& De Gelder, 2002). Four of the CP individuals included in our sample here participated in a series of fMRI studies, which mapped out the BOLD activation for faces, houses, objects, and patterns in relation to retinotopic meridians (Avidan et al., 2005) (the fifth subject could not be scanned). Not only was the time course of the activation normal for all $\mathrm{CP}$, but the location of activation was normal as well. Interestingly, one critical difference was observed in BOLD activity for faces and this was in prefrontal cortex, suggesting that the $\mathrm{CP}$ individuals might be taxing working memory more than normal subjects do, specifically when face processing is involved.

Although we have made considerable progress in addressing the issues we set out to investigate, many outstanding questions remain to be answered regarding $\mathrm{CP}$. Among them are the extent to which faces can be covertly or implicitly processed, the reason why the CP individuals have not developed compensatory mechanisms for their deficit at either a psychological or neural level and whether subtle anatomical or physiological differences might be able to account for $\mathrm{CP}$. These issues remain to be addressed in future research.

\section{Experimental Procedures}

\section{Subjects}

Five individuals with $\mathrm{CP}$, aged between 27 and 60 years of age, with no discernable cortical lesion or any history of neurological disease, participated. All were native English speakers, right-handed, and had normal or corrected-to-normal visual acuity (see Table 1). We tested a further four individuals (three of whom were members of the same family and one of whom was the mother of BE, one of the CP subjects), all of whom were self-declared congenitally prosopagnosic. Although the performance of these individuals was not entirely normal and they performed poorly on some of the face tests, they did not show unequivocal impairment across all tests and were thus excluded. For this study, we adopted very conservative inclusion criteria to ensure the purity of the sample, recognizing the potential for false alarms in self-report (De Haan, 1999).

Twelve control subjects, roughly matched to the CP individuals on age, sex, handedness, and education, were also recruited. Finally, three individuals with clearly defined AP following brain damage sustained in adulthood (age 16 in CR) were included. All three AP patients, SM, CR, and RN, have participated in previous studies and some of their data reported here have been published (for further details, see Behrmann, Marotta, Gauthier, Tarr, \& McKeeff, 2005; Behrmann \& Kimchi, 2003; Marotta, McKeeff, et al., 2002; Marotta, Behrmann, \& Genovese, 2001; Gauthier et al., 1999). This study was approved by the Institutional Review Boards of Carnegie Mellon University and of the University of Pittsburgh Medical Center, and written informed consent was obtained from all participants.

\section{METHODS}

Computer tasks were run on a Macintosh G3, using either ePrime or Matlab 5.2 at a viewing distance of approximately $65 \mathrm{~cm}$ from the screen. In all experiments, stimuli remained visible until the subject responded by pressing designated keys on the keyboard or by providing a verbal response. Instructions were to respond as quickly and accurately as possible and practice trials were given before each experiment.

\section{Face-related Tasks}

\section{Famous Faces Identification}

Subjects were shown two sets of famous faces. For the first set, 97 photographs were presented individually on a computer screen until subjects named each face, provided any other relevant information about the face (e.g., profession), or said they did not know anything about the face (Figure 1A). A response was considered correct if the correct name was provided. For the second set, 56 photographs were of famous individ- 
uals and the remaining 56 were unfamiliar (e.g., famous actors or celebrities from other countries) (Figure 1C). Photographs were chosen so as not to contain any salient or diagnostic cues. Roughly half the photographs were in color and half in monochrome. Half the faces were male and the other half female. Images were cropped to fit into a black oval to maintain equivalent size across pictures. Instructions were to name the person, provide any relevant contextual information, or report "don't know."

\section{Face Discrimination}

Subjects decided whether two faces were the same or different and pressed one of two keys to respond (Gauthier et al., 1999) (Figure 2A). Faces were paired in three conditions: (1) identical (20 trials), (2) different gender and individual (GI, 20 trials), and (3) same gender, different individual (I, 20 trials).

\section{Upright and Inverted Faces}

The stimuli consisted of 60 gray-scale faces (half male, half female) containing no diagnostic or salient cues, scanned from a 3Dlaser and obtained from Bülthoff and Troje (Max Planck Institute, Tübingen, Germany) (Figure 3A). All faces were cropped using the same $2.25 \times 3.00$-inch oval window to remove cues from the hairline and face contour. On each trial, a target (above) and two choices (lower right and left side) appeared on the screen and subjects indicated by button press which choice matched the target (Marotta, McKeeff, et al., 2002). The target and the two choices (always shown from the same view) could appear in the frontal, three-quarter, or profile view. In half the trials, the faces were upright and in the remaining half, they were inverted.

\section{Nonface-related Tasks}

\section{Object Discrimination}

Two stimuli appeared for a same/different judgment (Figure 4A). When they differed, they did so at either the basic (a chair and a duck), subordinate (a duck and a pelican), or exemplar level (2 different ducks), and there were 20 trials of each type.

\section{Greeble Discrimination}

In each trial, two Greebles (or a Greeble and an object) appeared on the screen for a same/different judgment (see Behrmann, Marotta, et al., 2005; Gauthier et al., 1999; Figure 5A). Stimuli could differ at a basic level (a Greeble and a car), or could both be Greebles but differ at the gender (different directions of appendages), family (different main bodies), or individual (same direction of appendages and main body but different shape appendages) level. There were 30 trials of each kind.

\section{Configural Tasks}

\section{Global/Local Perception}

This experiment examines the CP individuals' abilities to integrate local aspects of a display into a coherent global configuration (Figure 6A). The stimuli were four hierarchical letters of two types: consistent letters, in which the global and the local letters shared identity (a large $\mathrm{H}$ made of smaller Hs and a large S made of small Ss), and inconsistent letters in which the letters at the two levels had different identities (a large $\mathrm{H}$ made of small Ss and a large $\mathrm{S}$ made of small $\mathrm{Hs}$ ). The global letter subtended $3.2^{\circ}$ in height and $2.3^{\circ}$ in width, and the local letter subtended $0.44^{\circ}$ in height and $0.53^{\circ}$ in width. Subjects identified the letter at either the global or local level in separate blocks of trials in which consistent and inconsistent letters were randomized. Each block $(n=$ 96 trials) was preceded by instructions to identify at the local or global level. The order of the blocks and response was counterbalanced. A trial was initiated with a central fixation cross of 500-msec duration, which was immediately replaced by one of the four possible stimuli. Participants pressed one of two keys on the keyboard to indicate a response of "S" or "H."

\section{Microgenetic Analysis of Configural Organization}

This paradigm involved a prime followed by a test pair and subjects decided whether the two patterns of the test pair were the same or different. The priming stimuli and the same- and different-response test pairs are presented in Figure 7A. The priming stimuli were hierarchical patterns (global diamonds made up of circles) of two types: a few-element pattern and a many-element pattern, administered in separate blocks of 160 trials each. The few-element prime was a diamond made of 4 relatively large circles, and the many-element prime was a diamond made of 16 relatively small circles. Each test pair contained two hierarchical patterns. There were two types of test pairs defined by their prime-test similarity: the Element-Similarity (ES) test pairs in which the two figures were similar to the prime in their local elements but differed in global configuration, and the Configuration-Similarity (CS) test pairs in which the figures were similar to the prime in global configuration but differed in local elements. The global diamond subtended $1.25^{\circ}$ of visual angle, and the global square $0.96^{\circ}$. Each circle element subtended $0.36^{\circ}$ (in diameter) in the few-element and $0.18^{\circ}$ in the manyelement patterns. Each individual square element subtended $0.38^{\circ}$ in the few-element patterns and $0.19^{\circ}$ in the many-element patterns. The distance between the centers of the stimuli in a test pair was $7 \mathrm{~cm}$. 
All the combinations of the three factors (prime duration, test type, and response) were randomized within block with each combination occurring on an equal number of trials. On each trial, a small fixation dot appeared in the center of the screen for $250 \mathrm{msec}$, followed by a prime. The presentation time for the priming stimulus was equally and randomly distributed among 40, 90, 190, 390, and 690 msec. Immediately after the presentation of the prime, the test display appeared and stayed until the participant responded, for a maximum of $3000 \mathrm{msec}$. The two figures of the test pair appeared on either side of the location previously occupied by the prime and participants made the same/ different judgment by pressing one of two response keys. Each individual completed 320 trials with practice trials for few- and many-element patterns.

\section{Low-level Visual Testing}

To establish log contrast thresholds across the range of spatial frequencies from 1 to 30 cycles per inch (cpi), a pair of images was presented sequentially, one of which contained the frequency-modulated image and subjects performed a forced-choice discrimination of first versus second image (see Figure 8A). If the response was correct, a more difficult discrimination (decreased contrast by 0.2) was presented on the next trial. If the response was incorrect, the contrast was increased by 0.2. Each frequency bin $(0.3,1,3,10,30)$ was tested in a separate block and a log contrast threshold was determined for each cpi using method of limits where threshold is defined as the value of contrast that produces $82 \%$ accuracy in response.

To examine contour detection thresholds, we used cards containing a smoothly aligned closed path of Gabor elements, embedded in a random array of Gabor elements of the same spatial frequency and contrast (Pennefather, Chandna, Kovacs, Polat, \& Norcia, 1999) (Figure $8 \mathrm{~B}$ ). The cards are presented individually and the subject is required to indicate the location of the contour formed by the Gabor patches. The critical manipulation or parameter is the spacing between the adjacent elements in the background relative to the spacing between neighboring elements along the contour. $\Delta$ ranges between 1.15 (card 2_1) to 0.5 (card 2 15) in steps of 0.05 . This parameter expresses "relative noise density," and reflects signal-to-noise ratio so that the smaller the $\Delta$ value, the better detection. As $\Delta$ decreases, long-range spatial interactions of oriented features, presumably mediated by low-level areas of the visual cortex, are increasingly involved.

\section{Data Analysis}

In all experiments aside from the famous faces task and tasks of low-level vision, RT for correct trials and accuracy data were analyzed. Where possible, $A^{\prime}$ was also calculated. $A^{\prime}$ is a nonparametric measure of sensitivity, allowing us to characterize the discrimination performance of the subjects, independent of response bias. An $A^{\prime}$ of .5 indicates chance performance and more positive values indicate better than chance sensitivity. The $\mathrm{CP}$ group was always compared to the control subjects and, where possible, to the AP patients.

\section{Famous Faces}

The performance of the CP group was compared with that of the control group, separately for the two sets of famous faces. For the latter set, an ANOVA was conducted with group as the between-subjects factor and response (name correct, context correct, don't know, and incorrect) and number of trials as the dependent measures.

\section{Low-level Visual Tests}

Thresholds were established for each CP subject according to the procedures spelled out for each experiment.

\section{Acknowledgments}

We thank Byron Kohut, Erin Reeve, and Grace Lee Leonard for their assistance in data collection and analysis. Dr D. Giaschi (University of British Columbia) and Dr J. Janowski (Oregon Health and Science University) also collected some data for us and we thank them for this. We thank Heinrich Bülthoff for providing us with the MPI faces and Ilona Kovacs for the Gabor images. This study was funded by grants from the National Institutes of Mental Health to MB (MH54246), from the USIsrael Binational Science Foundation to $\mathrm{MB}$ and RK, and from the McDonnell Pew Program in Cognitive Neuroscience to JJM.

Reprint requests should be sent to Marlene Behrmann, Department of Psychology, Baker Hall, Carnegie Mellon University, 5000 Forbes Avenue, Pittsburgh, PA 15213-3890, USA, or via e-mail: behrmann@cnbc.cmu.edu.

\section{REFERENCES}

Ariel, R., \& Sadeh, M. (1996). Congenital visual agnosia and prosopagnosia in a child. Cortex, 32, 221-240.

Avidan, G., Hasson, U., Malach, R., \& Behrmann, M. (2005). Detailed exploration of face-related processing in congenital prosopagnosia: 2. Functional neuroimaging findings. Journal of Cognitive Neuroscience, 17, 1150-1167.

Bacon, W. F., \& Egeth, H. E. (1991). Local processes in pre-attentive feature detection. Journal of Experimental Psychology: Human Perception and Performance, 17, 77-90.

Badcock, C. J., Whitworth, F. A., Badcock, D. R., \& Lovegrove, W. J. (1990). Low-frequency filtering and processing of local-global stimuli. Perception, 19, 617-629.

Barton, J. J. S., Cherkasova, M. V., Press, D. Z., Intriligator, J. M., \& O'Connor, M. (2003). Developmental prosopagnosia: 
A study of three patients. Brain and Cognition, 51, 12-30.

Barton, J. J. S., Press, D. Z., Keenan, J. P., \& O’Connor, M. (2002). Lesions of the fusiform face area impair perception of facial configuration in prosopagnosia. Neurology, 58, $71-78$.

Barton, J. J. S., Zhao, J., \& Keenan, J. P. (2003). Perception of global facial geometry in the inversion effect and prosopagnosia. Neuropsychologia, 41, 1703-1711.

Behrmann, M., \& Avidan, G. (2005). Congenital prosopagnosia: Face-blind from birth. Trends in Cognitive Science, 9, 180-187.

Behrmann, M., Avidan, G., Lee, G., Kimchi, R., Luna, B., Humphreys, K., \& Minshew, N. (2005). Configural processing in autism and its relationship to face processing, Neuropsychologia.

Behrmann, M., \& Kimchi, R. (2003). What does visual agnosia tell us about perceptual organization and its relationship to object perception? Journal of Experimental Psychology: Human Perception and Performance, 29, 19-42.

Behrmann, M., Marotta, J., Gauthier, I., Tarr, M. J., \& McKeeff, T. J. (2005). Behavioral change and its neural correlates in prosopagnosia after expertise training. Journal of Cognitive Neuroscience, 17, 554-568.

Behrmann, M., \& Moscovitch, M. (2001). Face recognition: Evidence from intact and impaired performance. In F. Boller \& J. Grafman (Eds.), Handbook of neuropsychology (Vol. 4, pp. 181-206). North-Holland, Amsterdam: Elsevier.

Beller, H. K. (1971). Priming: effects of advance information on matching. Journal of Experimental Psychology, 87, 176-182.

Bentin, S., Allison, T., Puce, A., Perez, E., \& McCarthy, G. (1996). Electrophysiological studies of face perception in humans. Journal of Cognitive Neuroscience, 8 , 551-565.

Bentin, S., Deouell, L. Y., \& Soroker, N. (1999). Selective visual streaming in face recognition: Evidence from developmental prosopagnosia. NeuroReport, 10, 823-827.

Bruce, V., Valentine, T., \& Baddeley, A. (1987). The basis of the $3 / 4$ view advantage in face recognition. Applied Cognitive Psychology, 1, 109-120.

Bruce, V., \& Young, A. (1986). Understanding face recognition. British Journal of Psychology, 77, 305-327.

Carey, S., \& Diamond, R. (1994). Are faces perceived as configurations more by adults than by children? Visual Cognition, 1, 253-274.

Crawford, J. R., \& Garthwaite, P. H. (2002). Investigation of the single test score in neuropsychology: Confidence limits on the abnormality of test scores and test score differences. Neuropsychologia, 40, 1196-1208.

Damasio, A. R., Damasio, H., \& Hoesen, G. W. V. (1982). Prosopagnosia: Anatomic basis and behavioral mechanisms. Neurology, 32, 331-341.

de Gelder, B., Bachoud-Levi, A. C., \& Degos, J. D. (1998). Inversion superiority in visual agnosia may be common to a variety of orientation polarised objects besides faces. Vision Research, 38, 2855-2861.

de Gelder, B., \& Rouw, R. (2000). Configural face processes in acquired and developmental prosopagnosia: Evidence for two separate face systems? NeuroReport, 11, 3145-3150.

De Haan, E. H. F. (1999). A familial factor in the development of face recognition deficits. Journal of Clinical and Experimental Neuropsychology, 21, 312-315.

De Haan, E. H. F., \& Campbell, R. (1991). A fifteen year follow-up of a case of developmental prosopagnosia. Cortex, 27, 489-509.

De Renzi, E., Faglioni, P., Grossi, D., \& Nichelli, P. (1991).
Apperceptive and associative forms of prosopagnosia. Cortex, 27, 213-221.

Delvenne, J.-F., Seron, X., Coyette, F., \& Rossion, B. (2004). Evidence for perceptual deficits in associative visual (prosop)agnosia; a single case study. Neuropsychologia, 42, 597-612

Demonet, J. F., Taylor, M. J., \& Chaix, Y. (2004). Developmental dyslexia. Lancet, 363, 1451-1460.

Duchaine, B. C. (2000). Developmental prosopagnosia with normal configural processing. NeuroReport, 11, 79-83.

Duchaine, B. C., Dingle, K., Butterworth, E., \& Nakayama, K. (2004). Normal Greeble learning in a severe case of developmental prosopagnosia. Neuron, 43, 466-473.

Duchaine, B. C., \& Nakayama, K. (2004). Developmental prosopagnosia and the Benton Facial Recognition test. Neurology, 62, 1219-1220.

Duchaine, B., \& Nakayama, K. (2005). Dissociations in face and object recognition in developmental prosopagnosia. Journal of Cognitive Neuroscience, 17, 249-261.

Duchaine, B. C., Nieminen-von Wendt, T., New, J., \& Kulomaki, T. (2003). Dissociations of visual recognition in a developmental agnosic: Evidence for separate developmental processes. Neurocase, 9, 380-389.

Etcoff, N., Freeman, R., \& Cave, K. R. (1991). Can we lose memories of faces? Content specificity and awareness in a prosopagnosic. Journal of Cognitive Neuroscience, 3, 25-41.

Farah, M. J. (2004). Visual agnosia: Disorders of object recognition and what they tell us about normal vision (2nd ed.). Cambridge: MIT Press.

Farah, M. J., Rabinowitz, C., Quinn, G. E., \& Liu, G. T. (2000). Early commitment of neural substrates for face recognition. Cognitive Neuropsychology, 17, 117-123.

Farah, M. J., Wilson, K., Drain, H. M., \& Tanaka, J. R. (1998). What is "special" about face perception? Psychological Review, 105, 482-498.

Farah, M. J., Wilson, K. D., Drain, H. M., \& Tanaka, J. R. (1995). The inverted face inversion effect in prosopagnosia: Evidence for mandatory, face-specific perceptual mechanisms. Vision Research, 35, 2089-2093.

Francks, C., MacPhie, I. L., \& Monaco, A. P. (2002). The genetic basis of dyslexia. Lancet Neurology, 1, 483-490.

Freire, A., Lee, K., \& Symons, L. A. (2000). The face-inversion effect as a deficit in the encoding of configural information: Direct evidence. Perception, 29, 159-170.

Galaburda, A. M., \& Duchaine, B. C. (2003). Developmental disorders of vision. Neurologic Clinics, 21, 1-16.

Gauthier, I., Behrmann, M., \& Tarr, M. J. (1999). Can face recognition really be dissociated from object recognition? Journal of Cognitive Neuroscience, 11, 349-370.

Gauthier, I., Behrmann, M., \& Tarr, M. J. (2004). Are Greebles like faces? Using the neuropsychological exception to test the rule. Neuropsychologia, 42, 1961-1970.

Gauthier, I., \& Tarr, M. J. (2002). Unraveling mechanisms for expert object recognition: Bridging brain activity and behavior. Journal of Experimental Psychology: Human Perception and Performance, 28, 431-446.

Gerlach, C., Marstrand, L., Habekost, T., \& Gade, A. (in press). A case of impaired shape integration: Implications for models of visual object recognition. Visual Cognition.

Ginsburg, A. P. (1986). Spatial filtering and visual form information. In K. R. Boff, L. Kaufman, \& J. P. Thomas (Eds.), Handbook of human perception and performance (pp. 1-41). New York: Wiley.

Grill-Spector, K. (2003). The neural basis of object recognition. Current Opinion in Neurobiology, 13, 159-166. 
Grill-Spector, K., Knouf, N., \& Kanwisher N. (2004). The fusiform face area subserves face perception, not generic within-category identification. Nature Neuroscience, 7 , 555-562.

Grueter, M., Grueter, T., Bell, V., Horst, J., Laskowski, W., Sperling, K., Halligan, P. W., Ellis, H. D., \& Kennerknecht, I. (in press). Hereditary prosopagnosia: The first case series. Cortex.

Hadjikhani, N., \& De Gelder, B. (2002). Neural basis of prosopagnosia. Human Brain Mapping, 16, 176-182.

Hasson, U., Avidan, G., Deouell, L. Y., Bentin, S., \& Malach, R. (2003). Face-selective activation in a congenital prosopagnosic subject. Journal of Cognitive Neuroscience, 15, 419-431.

Haxby, J. V., Gobbini, M. I., Furey, M. L., Ishai, A., Schouten, J. L., \& Pietrini, P. (2001). Distributed and overlapping representations of faces and objects in ventral temporal cortex. Science, 293, 2425-2429.

Hill, H., Schyns, P. G., \& Akamatsu, S. (1997). Information and viewpoint dependence in face recognition. Cognition, 62, 201-222.

Hughes, H. C., Fendrich, R., \& Reuter-Lorenz, P. (1990). Global versus local processing in the absence of low spatial frequencies. Journal of Cognitive Neuroscience, 2, 272-282.

Humphreys, G. W. (1999). Integrative agnosia. In G. W. Humphreys (Ed.), Case studies in vision (pp. 41-58). London: Psychology Press.

Jones, R. D., \& Tranel, D. (2001). Severe developmental prosopagnosia in a child with superior intellect. Journal of Clinical and Experimental Neuropsychology, 23, 265-273.

Kanwisher, N. (2000). Domain specificity in face perception. Nature Neuroscience, 3, 759-763.

Kanwisher, N., McDermott, J., \& Chun, M. M. (1997). The fusiform face area: A module in human extrastriate cortex specialized for face perception. Journal of Neuroscience, 17, 4302-4311.

Kimchi, R. (1998). Uniform connectedness and grouping in the perceptual organization of hierarchical patterns. Journal of Experimental Psychology: Human Perception and Performance, 24, 1105-1118.

Kimchi, R. (2000). The perceptual organization of visual objects: A microgenetic analysis. Vision Research, 40, 1333-1347.

Kosslyn, S. M., Hamilton, S. E., \& Bernstein, J. H. (1995). The perception of curvature can be selectively disrupted in prosopagnosia. Brain and Cognition, 27, 36-58.

Kovács, I., Polat, U., Pennefather, P. M., Chandna, A., \& Norcia, A. M. (2000). A new test of contour integration deficits in patients with a history of disrupted binocular experience during visual development. Vision Research, 40, 1775-1783.

Kress, T., \& Daum, I. (2003a). Developmental prosopagnosia: A review. Behavioral Neurology, 14, 109-121.

Kress, T., \& Daum, I. (2003b). Event-related potentials reflect impaired face recognition in patients with congenital prosopagnosia. Neuroscience Letters, 352, 133-136.

Lamb, M., \& Yund, E. W. (1993). The role of spatial frequency in the processing of hierarchically organized structure. Perception and Psychophysics, 54, 773-784.

Leder, H., \& Bruce, V. (2000). When inverted faces are recognized: The role of configural information in face recognition. Quarterly Journal of Experimental Psychology A, 53, 513-536.

Le Grand, R., Mondloch, C. J., Maurer, D., \& Brent, H. P.
(2001). Neuroperception. Early visual experience and face processing. Nature, 410, 890.

Levine, D. N., \& Calvanio, R. (1989). Prosopagnosia: A defect in visual-configurational processing. Brain and Cognition, 10, $149-170$.

Marotta, J. J., Behrmann, M., \& Genovese, C. (2001). A functional MRI study of face recognition in patients with prosopagnosia. NeuroReport, 12, 959-965.

Marotta, J. J., McKeeff, T. J., \& Behrmann, M. (2002). The effects of inversion and rotation on face processing in prosopagnosia. Cognitive Neuropsychology, 19, 31-47.

Maurer, D., Le Grand, R., \& Mondloch, C. J. (2002). The many faces of configural processing. Trends in Cognitive Sciences, 6, 255-260.

McConachie, H. R. (1976). Developmental prosopagnosia: A single case report. Cortex, 12, 76-82.

McNeil, M., \& Warrington, E. K. (1993). Prosopagnosia: A face-specific disorder. Quarterly Journal of Experimental Psychology, 46A, 1-10.

Moscovitch, M., Winocur, G., \& Behrmann, M. (1997). What is special about face recognition? Nineteen experiments on a person with visual object agnosia and dyslexia but normal face recognition. Journal of Cognitive Neuroscience, 9, 555-604.

Moses, Y., Ullmann, S., \& Edelman, S. (1996). Generalization to novel images in upright and inverted faces. Perception, 25, 443-461.

Navon, D. (1977). Forest before trees: The precedence of global features in visual perception. Cognitive Psychology, 9, 353-383.

Navon, D. (2003). What does a compound letter tell the psychologist's mind? Acta Psychologica (Amsterdam), 114, 273-309.

Nunn, J. A., Postma, P., \& Pearson, R. (2001). Developmental prosopagnosia: Should it be taken at face value? Neurocase, 7, 15-27.

O’Toole, A. J., Edelman, S., \& Bulthoff, H. H. (1998). Stimulus-specific effects in face recognition over changes in viewpoint. Vision Research, 38, 2351-2363.

Palmer, S. E. (2003). Perceptual organization and grouping. In R. Kimchi, M. Behrmann, \& C. Olson (Eds.), Perceptual organization: Behavioral and neural processes (pp. 3-44). Mahwah, NJ: Erlbaum.

Pennefather, P. M., Chandna, A., Kovacs, I., Polat, U., \& Norcia, A. M. (1999). Contour detection threshold: repeatability and learning with "contour cards". Spatial Vision, 2, 257-266.

Peretz, I., \& Hyde, K. L. (2003). What is specific to music processing? Insights from congenital amusia. Trends in Cognitive Sciences, 7, 362-367.

Perrett, D. I., Rolls, E. T., \& Caan, W. (1979). Temporal lobe cells of the monkey with visual responses selective for faces. Neuroscience Letters, S3, S358.

Rhodes, G. (1988). Looking at faces: First-order and second-order features as determinates of facial appearance. Perception, 17, 43-63.

Riddoch, M. J., \& Humphreys, G. W. (1987). A case of integrative visual agnosia. Brain, 110, 1431-1462.

Rolls, E. T. (1984). Neurons in the cortex of the temporal lobe and in the amygdala of the monkey with responses selective for faces. Human Neurobiology, 3, 209-222.

Sekuler, A. B., \& Palmer, S. E. (1992). Perception of partly occluded objects: A microgenetic analysis. Journal of Experimental Psychology: General, 121, 95-111.

Sergent, J., \& Poncet, M. (1990). From covert to overt recognition of faces in a prosopagnosia patient. Brain, 113, 989-1094. 
Sergent, J., \& Signoret, J.-L. (1992). Functional and anatomical decomposition of face processing: Evidence from prosopagnosia and PET study of normal subjects. Philosophical Transactions of the Royal Society of London: B, 335, 55-62.

Shulman, G. L., Sullivan, M. A., Gish, K., \& Sakoda, W. J. (1986). The role of spatial-frequency channels in the perception of local and global structure. Perception, 15, 259-273.

Shulman, G. L., \& Wilson, J. (1987). Spatial frequency and selective attention to local and global information.

Neuropsychologia, 18, 89-101.

Tanaka, J. W., \& Farah, M. J. (1993). Parts and wholes in face recognition. Quarterly Journal of Experimental Psychology, 46A, 225-245.

Tarr, M. J., \& Cheng, Y. D. (2003). Learning to see faces and objects. Trends in Cognitive Neuroscience, 7, 23-30.

Tarr, M. J., \& Gauthier, I. (2000). FFA: A flexible fusiform face area for subordinate-level visual processing automatized by expertise. Nature Neuroscience, 3, 764-769.

Temple, C. M. (1992). Developmental memory impairment: Faces and patterns. In R. Campbell (Ed.), Mental lives: Case studies in cognition (pp. 199-215). Oxford: Blackwell. Yin, R. K. (1969). Looking at upside-down faces. Journal of Experimental Psychology, 81, 141-145. 